\title{
AN EXPLICIT FINITE-DIFFERENCE SCHEME FOR ONE-DIMENSIONAL GENERALIZED POROUS MEDIUM EQUATIONS: INTERFACE TRACKING AND THE HOLE FILLING PROBLEM
}

\author{
LÉONARD MONSAINGEON ${ }^{1}$
}

\begin{abstract}
Based on results of E. DiBenedetto and D. Hoff we propose an explicit finite-difference scheme for one dimensional Generalized Porous Medium Equations $\partial_{t} u=\partial_{x x}^{2} \Phi(u)$. The scheme allows to track the moving free boundaries, and captures the so-called hole filling phenomenon when free boundaries collide. We prove the convergence of the discrete solution when the mesh parameter $\Delta x \rightarrow 0$. Finally, we provide numerical evidence of the convergence of the scheme.
\end{abstract}

Mathematics Subject Classification. 65M06, 35R35, 35K65.

Received April 6, 2015. Revised July 11, 2015.

\section{INTRODUCTION}

We consider in this work the numerical approximation of nonnegative solutions $u(x, t) \geq 0$ to one-dimensional degenerate diffusion equations of the Generalized Porous Medium Equation type

$$
\partial_{t} u=\partial_{x x}^{2} \Phi(u), \quad t \geq 0, x \in \mathbb{R} .
$$

The nonlinearity $\Phi(s)$ is normalized as $\Phi(0)=0$, is monotone increasing for $s>0$, and satisfies the structural condition

$$
1<a \leq \frac{s \Phi^{\prime}(s)}{\Phi(s)} \leq b
$$

for some constants $a, b$. This roughly means that nonlinearities in the class $\Gamma_{a, b}$ behave in between two pure powers $s^{a}, s^{b}$ for $1<a \leq b$, which is a generalization of the celebrated Porous Medium Equation (PME) $\partial_{t} u=\Delta u^{m}$ for $m>1$. Moreover, $a>1$ implies that $\Phi(s) / s$ is monotone increasing and $\lim _{s \rightarrow 0^{+}} \frac{\Phi(s)}{s}=\Phi^{\prime}(0)=0$. Writing $\partial_{x x}^{2} \Phi(u)=\partial_{x}\left(\Phi^{\prime}(u) \partial_{x} u\right)$ the equation clearly degenerates at the levelset $\{u=0\}$, which results in the well known finite speed of propagation: if the initial data $u^{0}(x) \geq 0$ is compactly supported then $u(., t)$ remains compactly supported for all $t>0$, and free-boundaries $\Gamma(t)=\partial \operatorname{supp} u(., t)$ separate $\{u=0\}$ from $\{u>0\}$. Degenerate diffusion equations such as (GPME) have attracted considerable attention in the last decades. We refer the reader to $[8,9,13,19,22]$ and references therein for the Cauchy problem and regularity theory, and to $[2,5,6,10,11]$ for the theory of free-boundaries.

\footnotetext{
Keywords and phrases. Generalized porous medium equation, interface tracking, hole filling, finite-difference.

1 CAMGSD, Instituto Superior Técnico, Av. Rovisco Pais, 1049-001 Lisboa, Portugal. leonard.monsaingeon@ist.utl.pt
} 
In order to understand the propagation of free-boundaries it is more convenient to use the pressure variable

$$
v:=\Psi(u), \quad \Psi(s):=\int_{0}^{s} \frac{\Phi^{\prime}(z)}{z} \mathrm{~d} z,
$$

which formally solves

$$
v_{t}=\sigma(v) \partial_{x x}^{2} v+\left|\partial_{x} v\right|^{2}
$$

with

$$
\sigma(v):=\Phi^{\prime}(u)=\Phi^{\prime} \circ \Psi^{-1}(v) .
$$

The structural assumption (H) implies that $(a-1) v \leq \sigma(v) \leq(b-1) v$, and $v, \sigma(v), \Phi^{\prime}(u), \Phi(u) / u$ are comparable in the sense that the ratio of any two of these quantities is bounded away from zero and from above. In space dimension one the free-boundaries $\Gamma(t)=\partial \operatorname{supp} u(., t)=\partial \operatorname{supp} v(., t)$ can be simply parametrized by a set of curves $x=\zeta(t)$ and are usually referred to as interfaces. By definition along these curves $v=\sigma(v)=0$, thus formally discarding the $\sigma(v) \partial_{x x}^{2} v$ term in (1.1) we see that $\partial_{t} v=\left|\partial_{x} v\right|^{2}$ at the interfaces. This suggests that the motion of the free-boundaries is governed by $\mathrm{d} \zeta / \mathrm{d} t=-\partial_{x} v(\zeta(t), t)$ provided that these quantities exist in some sense, see e.g. $[5,11]$.

In order to track the interfaces we shall work exclusively in the pressure framework (1.1) rather than with (GPME), and we restrict in the whole paper to Lipschitz-continuous and compactly supported initial data

$$
0 \leq v^{0}(x) \leq M, \quad \operatorname{Lip}\left(v^{0}\right) \leq \gamma_{0} .
$$

Because (GPME) and (1.1) satisfy a comparison principle [22] we expect that $0 \leq v(x, t) \leq M$ for all times, and the behaviour of $\Phi(s)$ should therefore be irrelevant for large $r=\Psi(s) \geq M$. As a consequence we $\operatorname{relax}\left(\Gamma_{a, b}\right)$ and only assume throughout the whole paper

$$
\sigma \in \mathcal{C}^{1}\left([0, \infty), \mathbb{R}^{+}\right) \cap \mathcal{C}^{2}\left(\mathbb{R}^{+}, \mathbb{R}^{+}\right), \quad \sigma(0)=0, \quad \sigma^{\prime}>0,
$$

and

$$
\forall r \in[0, M]: \quad 0<s_{1}(M) \leq \sigma^{\prime}(r) \leq S_{1}(M) \quad \text { and } \quad\left|\sigma^{\prime \prime}(r)\right| \leq S_{2}(M)
$$

for structural $s_{1}, S_{1}, S_{2}$.

Remark 1.1. These conditions on $\sigma(r)$ can be translated into conditions on the original $\Phi(s)$ nonlinearity through $r=\Psi(s)$, for example $\sigma^{\prime}(r)=s \Phi^{\prime \prime}(s) / \Phi^{\prime}(s)$. In the case of the pure PME nonlinearity $\Phi(s)=s^{m}$ one can compute explicitly $v=\Psi(u)=m u^{m-1} /(m-1)$ and $\sigma(v)=(m-1) v$, thus $s_{1}=S_{1}=(m-1)$ and $S_{2}=0$ in $(\mathrm{H})$. As a consequence the above structural assumptions for $\sigma$ can be viewed as some PME-like behaviour condition in bounded intervals.

Because of gradient jumps at the free-boundaries no classical solutions can exist if $v^{0}$ has compact support, and we shall use the following weak formulation:

Definition 1.2. A function $0 \leq v \in \mathcal{C}(\mathbb{R} \times[0, T])$ is a weak solution of (1.1) with initial datum $v^{0}(x)$ if $\partial_{x} v \in L^{2}(\mathbb{R} \times(0, T))$ and

$$
\int_{\mathbb{R}} v(x, \tau) \varphi(x, \tau) \mathrm{d} x-\int_{\mathbb{R}} v^{0}(x) \varphi(x, 0) \mathrm{d} x+\int_{0}^{\tau} \int_{\mathbb{R}}\left\{-v \partial_{t} \varphi+\sigma(v) \partial_{x} v \cdot \partial_{x} \varphi+\left(1-\sigma^{\prime}(v)\right)\left|\partial_{x} v\right|^{2} \varphi\right\} \mathrm{d} x \mathrm{~d} t=0
$$

for all $0 \leq \tau \leq T$ and test functions $\varphi \in \mathcal{C}_{c}^{\infty}(\mathbb{R} \times[0, T])$.

Note that we impose here continuity at $t=0^{+}$, so that the initial data are taken in a strong sense. The equivalence between the density $u$ and pressure $v$ formulations with $v=\Psi(u)$ is well known [3], and any 
solution $v$ in the sense of Definition 1.2 corresponds to a weak solution $u=\Psi^{-1}(v)$ of (GPME). As we shall only work in the pressure variable we refrain from giving a precise definition of weak solutions for (GPME) and refer the reader e.g. to $[11,22]$.

The problem of numerical approximation to (1.1) in dimension one goes back to [16], where a finite-difference approach was first proposed to compute numerical solutions of $\partial_{t} v=f(x, t, v) \partial_{x x}^{2} v+\left|\partial_{x} v\right|^{2}$ but free-boundaries were not accurately tracked. Later in [20] a scheme allowing to track the interfaces was implemented for the pure PME nonlinearity $\Phi(s)=s^{m}$, but the authors were not able to prove convergence of the interface curves. Almost simultaneously, DiBenedetto and Hoff proposed in [14] an explicit finite-difference interface-tracking algorithm for the pure PME nonlinearity, and established rigorous error estimates for the solution and interfaces. In $[14,16,20]$ only the case of initial data $v^{0}$ consisting in a single patch is considered, i.e. the initial support is always assumed to have a single connected component $\operatorname{supp} v^{0}=\left[\zeta_{l}(0), \zeta_{r}(0)\right]$. In this case the interfaces can be represented by two continuous left/right curves $\zeta_{l r}(t)$ with $\operatorname{supp} v(., t)=\left[\zeta_{l}(t), \zeta_{r}(t)\right]$ for all $t \geq 0$. Due to the diffusive nature of the problem $\operatorname{supp} v(., t)$ is noncontracting in time ([11], Cor. 1.5), and as a consequence $\zeta_{l}(t)$ and $\zeta_{r}(t)$ are monotone nonincreasing and nondecreasing respectively. In addition to this simple setting we shall also consider here the so-called hole-filling problem when the initial support has an internal hole, i-e when $\operatorname{supp} v^{0}$ has an two connected components at positive distance from each other. Because of the finite speed of propagation the hole persists for small times, but eventually fills in finite time (see Sect. 3 for a detailed description of the problem). A finite-element method was recently employed in [23] to investigate the holefilling and related problems with satisfactory qualitative results, but without any rigorous convergence result. In the presence of absorption, the opposite support splitting phenomenon was numerically investigated in [18], see also [21] for a brief review. Finally, a finite difference was recently proposed [12] for a nonlocal version $\partial_{t} u+(-\Delta)^{\sigma / 2} u^{m}=0$ of the PME.

Closely following [14] we propose an extension of DiBenedetto and Hoff's algorithm to general nonlinearities, allowing to track the interfaces and solve past the hole-filling time. As in [14] the algorithm reproduces at the discrete level all the properties satisfied by the continuous solutions of (1.1). More precisely: initial $\gamma_{0}$ Lipschitz regularity, nonnegativity, and $L^{\infty}$ bounds are preserved along the time evolution, solutions are $1 / 2$ Hölder continuous in time, and satisfy a generalized Aronson-Bénilan estimate $\partial_{x x} v(x, t) \geq \underline{z}(t) \approx-C(1+1 / t)$ in the sense of distributions $\mathcal{D}^{\prime}\left(\mathbb{R}_{x}\right)$ for all fixed $t>0$. For the pure PME nonlinearity $\Phi(s)=s^{m}$ the latter semi-convexity property was first proved in [4] in the optimal form $\partial_{x x} v(x, t) \geq-1 /(m+1) t$, and is fundamental for the regularity and propagation theories. The numerical scheme is based on the following splitting method: inside the support $\{v>0\}=\{\sigma(v)>0\}$ (1.1) is formally parabolic, hence a classical finite-difference scheme can be used with an extra $\varepsilon$-viscosity stabilizing term. As already discussed the free-boundaries $x=\zeta(t)$ should evolve according to $\mathrm{d} \zeta / \mathrm{d} t=-\partial_{x} v$, and thus enforcing the discrete equivalent allows to track the interfaces. Technically speaking this propagation law will in fact be applied at the discrete level in some neighborhood of the interfaces. This neighborhood turns to have thickness of the same order $\mathcal{O}(\Delta x)$ as the mesh size $\Delta x$, and can therefore be viewed as a numerical boundary layer.

The paper is organized as follows: in Section 2 we describe the scheme for general nonlinearities when the initial data consists in a single patch. Imposing a suitable stability condition $\Delta t=\mathcal{O}\left(\Delta x^{2}\right)$ on the mesh parameters we establish discrete a priori bounds, including a generalized Aronson-Bénilan estimate (Lem. 2.5). These a priori estimates then allow us to prove convergence of the approximate solutions and interface curves when $\Delta x \rightarrow 0$. In Section 3 we extend the scheme to study the hole-filling problem, when internal interfaces collide in finite time. We construct a numerical approximation to the hole-filling time and show that our scheme really captures the hole-filling phenomenon, in the sense that it allows to keep computing a consistent approximation to the solution past the filling time. An abstract convergence result is given. In Section 4 we present numerical experiments and investigate the rates of convergence. We would like to stress that Section 2 is adapted from [14] to general nonlinearities but requires significant technical modifications, in particular for the generalized Aronson-Bénilan estimate (Lem. 2.5). To the best of our knowledge all the results in Section 3 are new, even for the pure PME nonlinearity. 


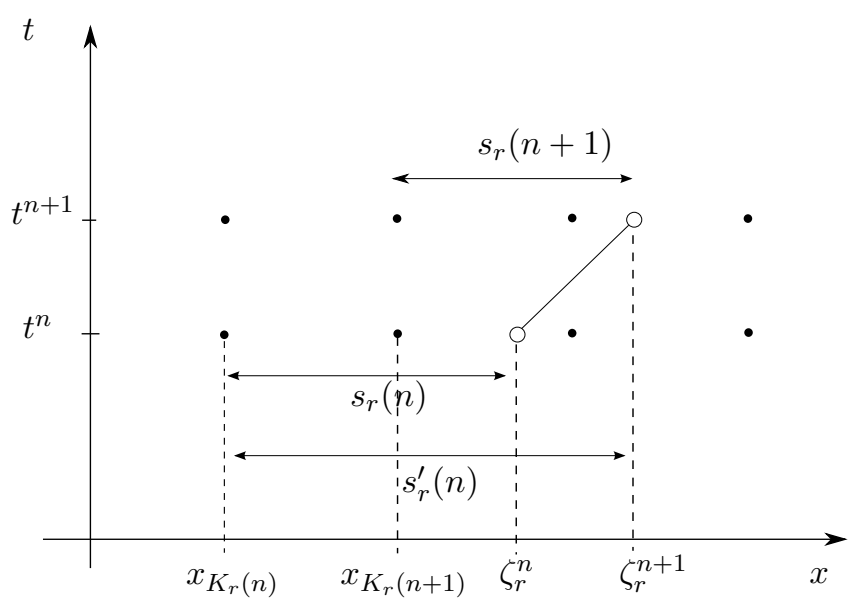

FiguRE 1. Right numerical boundary layer.

\section{THE SCHEME FOR ONE PATCH ONLY}

Throughout the whole paper we fix mesh parameters $\Delta x, \Delta t$ and write $\left\{x_{k}\right\}_{k \in \mathbb{Z}}=\{k \Delta x\},\left\{t^{n}\right\}_{n \geq 0}=\{n \Delta t\}$, as well as $v_{k}^{n} \approx v\left(x_{k}, t^{n}\right)$ and $\zeta_{l r}^{n} \approx \zeta_{l r}\left(t^{n}\right)$. Given a "single patch" compactly supported initial datum $v^{0}$

$$
0 \leq v^{0}(x) \leq M, \quad \operatorname{Lip}\left(v^{0}\right) \leq \gamma_{0}, \quad \operatorname{supp} v^{0}=\left[\zeta_{l}(0), \zeta_{r}(0)\right],
$$

we first initialize

$$
v_{k}^{0}:=v^{0}\left(x_{k}\right) \quad \text { and } \quad \zeta_{l, r}^{0}:=\zeta_{l, r}(0) .
$$

Given an approximate solution $v_{k}^{n}$ and interfaces $\zeta_{l, r}^{n}$ at time $t^{n}$, we define

$$
K_{l}(n):=\min \left\{k \in \mathbb{Z}: x_{k-1} \geq \zeta_{l}^{n}\right\}, \quad K_{r}(n):=\max \left\{k \in \mathbb{Z}: x_{k+1} \leq \zeta_{r}^{n}\right\}
$$

and

$$
0 \leq s_{l}(n):=x_{K_{l}(n)}-\zeta_{l}^{n}, \quad 0 \leq s_{r}(n):=\zeta_{r}^{n}-x_{K_{r}(n)} .
$$

We shall often speak of $x_{k} \in\left[x_{K_{l}(n)}, x_{K_{r}(n)}\right]$ as the (numerical) support at time $t^{n}$, while $x_{k} \in\left[\zeta_{l}^{n}, x_{K_{l}(n)}\right]$ and $x_{k} \in\left[x_{K_{r}(n)}, \zeta_{r}^{n},\right]$ will be referred to as the (numerical) left and right boundary layers. Observe that by construction these boundary layers have thickness $\Delta x \leq s(n) \leq 2 \Delta x$, see Figure 1 . The interfaces at time $t^{n+1}$ are next computed as

$$
\frac{\zeta_{l}^{n+1}-\zeta_{l}^{n}}{\Delta t}=-\frac{v_{K_{l}(n)}^{n}}{s_{l}^{n}}, \quad \frac{\zeta_{r}^{n+1}-\zeta_{r}^{n}}{\Delta t}=-\frac{v_{K_{r}(n)}^{n}}{s_{r}^{n}},
$$

thus reproducing the propagation law $\mathrm{d} \zeta / \mathrm{d} t=-\partial_{x} v$ at the free-boundaries. We will prove in Lemma 2.2 that $v_{k}^{n} \geq 0$, and therefore $\zeta_{l}^{n+1} \leq \zeta_{l}^{n}$ and $\zeta_{r}^{n+1} \geq \zeta_{r}^{n}$. This monotonicity translates the noncontractivity of the support at the discrete level. We also define for later use

$$
s_{l}^{\prime}(n):=x_{K_{l}(n)}-\zeta_{l}^{n+1} \geq s_{l}^{n}, \quad s_{r}^{\prime}(n):=\zeta_{r}^{n+1}-x_{K_{r}(n)} \geq s_{r}^{n} .
$$

Carefully note that $s_{l r}^{\prime}(n) \neq s_{l r}^{n+1}$ and that $x=\zeta_{l r}^{n}$ need not be integer meshpoints, see Figure 1 . The solution $v_{k}^{n+1}$ is then updated inside the support by enforcing

$$
k \in\left[K_{l}(n), K_{r}(n)\right]: \quad \frac{v_{k}^{n+1}-v_{k}^{n}}{\Delta t}=\left(\sigma\left(v_{k}^{n}\right)+\varepsilon\right) \frac{v_{k-1}^{n}-2 v_{k}^{n}+v_{k+1}^{n}}{\Delta x^{2}}+\left|\frac{v_{k+1}^{n}-v_{k-1}^{n}}{2 \Delta x}\right|^{2},
$$


where $\varepsilon>0$ is a fixed artificial viscosity parameter to be chosen later. Observe that (2.2) is not applied across the interfaces but only inside the numerical support, where (1.1) is formally in the parabolic regime since $\{v>0\}=\{\sigma(v)>0\}$.

Inside the boundary layers of thickness $s^{\prime}(n)$ the solution is linearly interpolated as

$$
v_{k}^{n+1}:=\left\{\begin{array}{cc}
v_{K_{l}(n) \frac{x_{k}-\zeta_{l}^{n+1}}{x_{K_{l}(n)}-\zeta_{l}^{n+1}}} & x_{k} \in\left[\zeta_{l}^{n+1}, x_{K_{l}(n)-1}\right] \\
v_{K_{r}(n) \frac{\zeta_{r}^{n+1}-x_{k}}{\zeta_{r}^{n+1}-x_{K_{r}(n)}}} & x_{k} \in\left[x_{K_{r}(n)+1}, \zeta_{r}^{n+1}\right]
\end{array},\right.
$$

and finally we set

$$
v_{k}^{n+1}:=0 \quad \text { for } x_{k} \notin\left[\zeta_{l}^{n+1}, \zeta_{r}^{n+1}\right] .
$$

The interpolation (2.3) is consistent with the well known linear behaviour of the pressure variable across the moving free boundaries ([22], Thm. 15.24) see Lemma 2.11 later on.

Remark 2.1. According to (2.3) $v_{k}^{n}$ is exactly linear in $x$ inside the boundary layers. As a consequence $(2.1)$ also reads $\frac{\zeta_{l}^{n+1}-\zeta_{l}^{n}}{\Delta t}=-\frac{v_{K_{l}(n)}-v_{K_{l}(n)-1}}{\Delta x}$ and $\frac{\zeta_{r}^{n+1}-\zeta_{r}^{n}}{\Delta t}=-\frac{v_{K_{r}(n)+1}-v_{K_{r}(n)}}{\Delta x}$, perhaps illustrating $\mathrm{d} \zeta / \mathrm{d} t=-\partial_{x} v$ even more clearly than $(2.1)$.

Throughout the whole paper and without further mention we impose the Courant-Fredrichs-Lewis stability condition

$$
\begin{aligned}
\frac{\Delta t}{\Delta x^{2}}:= & \beta \leq \frac{1}{2(\sigma(M)+\varepsilon)+\gamma_{0} \Delta x\left(4+3 S_{1}(M)\right)+\gamma_{0}^{2} \Delta x^{2} S_{2}(M) / 2} \\
& \gamma_{0} \Delta x\left(27+9 s_{1}(M)+3 S_{1}(M)+\Delta x S_{2}(M) / 4\right) \leq \varepsilon \leq \mathcal{O}(\Delta x)
\end{aligned}
$$

with $\left\|v^{0}\right\|_{L^{\infty}(\mathbb{R})} \leq M, \operatorname{Lip}\left(v^{0}\right) \leq \gamma_{0}$, and $s_{1}(M), S_{1}(M), S_{2}(M) \geq 0$ as in $(\mathrm{H})$.

\subsection{A priori discrete estimates}

Defining the discrete backward and centered derivatives

$$
w_{k}^{n}:=\frac{v_{k}^{n}-v_{k-1}^{n}}{\Delta x}, \quad \bar{w}_{k}^{n}:=\frac{v_{k+1}^{n}-v_{k-1}^{n}}{2 \Delta x},
$$

the first discrete estimate reads

Lemma 2.2. Assume that $0 \leq v_{k}^{0} \leq M$ with $\left|w_{k}^{0}\right| \leq \gamma_{0}$. Then for all $k, n$ there holds

$$
0 \leq v_{k}^{n} \leq M \quad \text { and } \quad\left|w_{k}^{n}\right| \leq \gamma_{0} .
$$

Proof. We write $\beta=\Delta t / \Delta x^{2}$, abbreviate $\sigma_{k}^{n}:=\sigma\left(v_{k}^{n}\right)$, and argue by induction on $n \geq 0$.

Step 1: Positivity and $l^{\infty}$ stability. Noting that $\frac{v_{k+1}^{n}-v_{k-1}^{n}}{2 \Delta x}=\frac{w_{k+1}^{n}+w_{k}^{n}}{2}$ it is easy to rewrite (2.2) inside the support $x_{k} \in\left[x_{K_{l}(n)}, x_{K_{r}(N)}\right]$ as

$$
v_{k}^{n+1}=(1-2 a) v_{k}^{n}+(a-b) v_{k-1}^{n}+(a+b) v_{k-1}^{n}
$$

with

$$
a:=\beta\left(\sigma_{k}^{n}+\varepsilon\right) \quad \text { and } \quad b:=\beta \Delta x\left(w_{k+1}^{n}+w_{k}^{n}\right) / 4 .
$$


By the induction hypothesis and monotonicity of $\sigma$ the (CFL) condition implies

$$
0 \leq \beta \varepsilon \leq a \leq \beta(\sigma(M)+\varepsilon) \leq \frac{1}{2}, \quad|b| \leq \Delta x \beta \gamma_{0} / 2 \leq \beta \varepsilon \leq a,
$$

thus $v_{k}^{n+1}$ is a convex combination of $v_{k-1}^{n}, v_{k}^{n}, v_{k+1}^{n} \in[0, M]$. In particular $0 \leq v_{k}^{n+1} \leq M$ for $k \in\left[K_{l}(n), K_{r}(n)\right]$, and by (2.3) clearly $0 \leq v_{k}^{n+1} \leq M$ for all $k$.

Step 2: Lischitz bounds in the support. Consider any $k \in\left[K_{l}(n)+1, K_{r}(n)\right]$, so that $v_{k}^{n+1}, v_{k-1}^{n+1}$ are both computed using (2.2) or equivalently

$$
v_{k}^{n+1}=v_{k}^{n}+\beta \Delta x\left(\sigma_{k}^{n}+\varepsilon\right)\left(w_{k+1}^{n}-w_{k}^{n}\right)+\beta \Delta x^{2} / 4\left(w_{k+1}^{n}+w_{k-1}^{n}\right)^{2} .
$$

Subtracting the corresponding equation for $v_{k-1}^{n+1}$ and dividing by $\Delta x$, straightforward manipulations lead to

$$
\begin{aligned}
w_{k}^{n+1}= & w_{k}^{n}+\Delta t\left[\left(\frac{\sigma_{k}^{n}+\sigma_{k-1}^{n}}{2}+\varepsilon\right) \frac{w_{k+1}^{n}-2 w_{k}^{n}+w_{k-1}^{n}}{\Delta x^{2}}\right. \\
& \left.+\left(\mathfrak{S}_{v} w_{k}^{n}+2 \frac{w_{k+1}^{n}+2 w_{k}^{n}+w_{k-1}^{n}}{4}\right) \frac{w_{k+1}^{n}-w_{k-1}^{n}}{2 \Delta x}\right]
\end{aligned}
$$

with

$$
\mathfrak{S}_{v}:=\frac{\sigma_{k}^{n}-\sigma_{k-1}^{n}}{v_{k}^{n}-v_{k-1}^{n}}=\frac{\sigma\left(v_{k}^{n}\right)-\sigma\left(v_{k-1}^{n}\right)}{v_{k}^{n}-v_{k-1}^{n}} \approx \sigma^{\prime}\left(v\left(x_{k}, t^{n}\right)\right) .
$$

Formula (2.6) is the discrete equivalent of

$$
w=\partial_{x} v: \quad \partial_{t} w=\sigma(v) \partial_{x x}^{2} w+\left[\sigma^{\prime}(v) w+2 w\right] \partial_{x} w,
$$

which is formally obtained differentiating (1.1) w.r.t. $x$. Considering (2.7) as a linear parabolic equation $\partial_{t} w=$ $a \partial_{x x}^{2} v+b \partial_{x} w$ with no zeroth order coefficient, we see that $w=\partial_{x} v$ formally satisfies the maximum principle. Thus the initial $\gamma_{0}$-Lipschitz bounds for $v^{0}$ should be preserved for $t \geq 0$ as in our statement.

In order to make this maximum principle rigorous at the discrete level we rewrite (2.6) as

$$
w_{k}^{n+1}=(1-2 a) w_{k}^{n}+(a-b) w_{k-1}^{n}+(a+b) w_{k+1}^{n},
$$

with now

$$
a=\beta\left(\frac{\sigma_{k}^{n}+\sigma_{k-1}^{n}}{2}+\varepsilon\right) \quad \text { and } \quad b=\beta\left(\frac{\mathfrak{S}_{v}}{2} w_{k}^{n}+\frac{w_{k+1}^{n}+2 w_{k}^{n}+w_{k-1}^{n}}{4}\right) \Delta x .
$$

By the induction hypothesis $0 \leq v_{k}^{n} \leq M$ and the structural assumption (H) we get $0 \leq \mathfrak{S}_{v} \approx \sigma^{\prime}\left(v_{k}^{n}\right) \leq S_{1}(M)$, and the (CFL) condition implies

$$
0 \leq \beta \varepsilon \leq a \leq \beta(\sigma(M)+\varepsilon) \leq 1 / 2 \quad \text { and } \quad|b| \leq \beta \gamma_{0}\left(\frac{S_{1}(M)}{2}+1\right) \Delta x \leq \beta \varepsilon \leq a .
$$

From (2.8) we see that $w_{k}^{n+1}$ is a convex combination of $w_{k-1}^{n}, w_{k-1}^{n}, w_{k+1}^{n}$ and we conclude that $\left|w_{k}^{n+1}\right| \leq \gamma_{0}$ as claimed.

Step 3: Lischitz bounds close to the interfaces. The computations at the left and right interfaces are identical, so we only deal with the right one and write $K=K_{r}(n), s(n)=s_{r}(n)=\zeta_{r}^{n}-x_{K}$ and $s^{\prime}(n)=$ $s_{r}^{\prime}(n)=\zeta_{r}^{n+1}-x_{K}$ for simplicity. By construction of the scheme $v_{k}^{n+1}$ is linear for $x_{k} \in\left[x_{K}, \zeta^{n+1}\right]$ and zero 
for $x_{k} \geq \zeta^{n+1}$. In particular $w_{K+1}^{n+1} \leq w_{k}^{n+1} \leq 0$ for all $x_{k} \geq x_{K+1}$ and it is clearly enough to estimate $\left|w_{K+1}^{n+1}\right|$. From (2.3) we see that $w_{K+1}^{n+1}=-\frac{v_{K}^{n+1}}{s^{\prime}(n)}$, and exploiting (2.2) we get

$$
\begin{aligned}
w_{K+1}^{n+1}= & -\frac{1}{s^{\prime}(n)}\left[v_{K}^{n}+\Delta t\left(\sigma_{K}^{n}+\varepsilon\right) \frac{v_{K+1}^{n}-2 v_{K}^{n}+v_{K_{1}}^{n}}{\Delta x^{2}}+\Delta t\left(\frac{v_{K+1}^{n}-v_{K-1}^{n}}{2 \Delta x}\right)^{2}\right] \\
= & -\frac{1}{s^{\prime}(n)}\left[v_{K}^{n}+\beta \Delta x\left(\sigma_{K}^{n}+\varepsilon\right)\left(w_{K+1}^{n}-w_{K}^{n}\right)\right. \\
& \left.+\Delta t\left\{\left(w_{K+1}^{n}\right)^{2}-\frac{w_{K}^{n}+3 w_{K+1}^{n}}{4}\left(w_{K+1}^{n}-w_{K}^{n}\right)\right\}\right] .
\end{aligned}
$$

According to Remark 2.1 we have $s^{\prime}(n)-s^{n}=\zeta^{n+1}-\zeta^{n}=-w_{K+1}^{n} \Delta t$, and since $v_{K}^{n}=-w_{K+1}^{n} s^{n}$ we get $v_{K}^{n}+\Delta t\left(w_{K+1}^{n}\right)^{2}=-w_{K+1}^{n} s^{\prime}(n)$. Substituting in the previous expression gives

$$
w_{K+1}^{n+1}=w_{K+1}^{n}+c\left(w_{K}^{n}-w_{K+1}^{n}\right)
$$

with

$$
c=\frac{\beta \Delta x}{s^{\prime}(n)}\left(\left(\sigma_{K}^{n}+\varepsilon\right)-\Delta x \frac{3 w_{K+1}^{n}+w_{K}^{n}}{4}\right) .
$$

Using the induction hypothesis, the (CFL) condition, and $s^{\prime}(n) \geq s(n) \geq \Delta x$ yields

$$
0 \leq \beta \frac{\Delta x}{s^{\prime}(n)}\left(\varepsilon-\gamma_{0} \Delta x\right) \leq c \leq \beta\left(\sigma(M)+\varepsilon+\gamma_{0} \Delta x\right) \leq 1,
$$

thus by (2.10) $\left|w_{K+1}^{n+1}\right| \leq \gamma_{0}$ as the convex combination of $w_{K}^{n}, w_{K+1}^{n}$ and the proof is complete.

As a consequence the interfaces propagate with finite speed:

Lemma 2.3. For all $t^{n} \geq 0$ there holds $\left|\frac{\zeta_{l r}^{n+1}-\zeta_{l r}^{n}}{\Delta t}\right| \leq \gamma_{0}$ and

$$
\zeta_{l}(0)-\gamma_{0} t^{n} \leq \zeta_{l}^{n} \leq \zeta_{l}(0) \leq \zeta_{r}(0) \leq \zeta_{r}^{n} \leq \zeta_{r}(0)+\gamma_{0} t^{n}
$$

Proof. By Remark $2.1\left|\left(\zeta^{n+1}-\zeta^{n}\right) / \Delta t\right|=\left|-w_{K(n) \pm 1}^{n}\right|$ so our statement immediately follows by Lemma 2.2 and the pinning $\zeta_{l n}^{0}=\zeta_{l r}(0)$. The monotonicity is a consequence of (2.1) with $v_{k}^{n} \geq 0$.

In the next auxiliary lemma we construct the lower bound to be used in the generalized Aronson-Bénilan estimate $\partial_{x x}^{2} v \geq \underline{z}(t)$ by means of a certain ODE:

Lemma 2.4. Let $\Lambda:=\gamma_{0}^{2} S_{2}(M)$ and $F(z):=\Lambda z+\left(2+s_{1}(M)\right) z^{2}$ with $s_{1}, S_{2}$ as in $(\mathrm{H})$. There is a function $\underline{z}(t): \mathbb{R}^{+} \rightarrow \mathbb{R}$ such that $\frac{d \underline{z}}{\mathrm{~d} t}=F(\underline{z})$ with $\lim _{t \backslash 0} \underline{z}(t)=-\infty$. Moreover $\underline{z}$ is monotone increasing and concave, $\underline{z}(t) \leq \underline{z}(\infty)=-\Lambda /\left(2+s_{1}(M)\right)$, and $\underline{z}(t) \sim-\frac{1}{\left(2+s_{1}(M)\right) t}$ when $t \searrow 0$.

Proof. Observe that $F(z)$ is a quadratic polynomial with $F\left(-\Lambda /\left(2+s_{1}(M)\right)\right)=0$. Picking any $t_{0}>0, z_{0}<$ $-\Lambda /\left(2+s_{1}(M)\right)$ and solving $\mathrm{d} z / \mathrm{d} t=F(z)$ with $z\left(t_{0}\right)=z_{0}$ it is easy to see that $z$ is monotone increasing in $(\underline{T}, \infty)$ with blow-up in finite time $z(\underline{T})=-\infty$ and $z(\infty)=-\Lambda /\left(2+s_{1}(M)\right)$. Shifting $\underline{z}(t):=z(t+\underline{T})$ gives the sought solution, and all the qualitative properties follow from a straightforward phase portrait analysis.

The generalized Aronson-Bénilan estimate then takes the form. 
Lemma 2.5. Let $\underline{z}(t)$ as in Lemma 2.4. Then for all $k, n$ there holds

$$
Z_{k}^{n}:=\frac{A v_{k}^{n}}{\Delta x^{2}}=\frac{v_{k-1}^{n}-2 v_{k}^{n}+v_{k+1}^{n}}{\Delta x^{2}} \geq \underline{z}\left(t^{n}\right) .
$$

Proof. Since $\underline{z}$ is monotone increasing with $\underline{z}(0)=-\infty$ the time $t^{N}=\max \left\{t^{n}: \underline{z}\left(t^{n}\right) \leq-2 \gamma_{0} / \Delta x\right\}$ is well defined and positive, provided that $\Delta x, \Delta t$ are small enough. By Lemma 2.2 we have $Z_{k}^{n}=\frac{w_{k+1}^{n}-w_{k}^{n}}{\Delta x} \geq-2 \gamma_{0} / \Delta x$ and our estimate automatically holds if $t_{n} \leq t^{N}$. We argue now by induction on $n \geq N$.

Step 1: Estimate in the support. Consider first any $k \in\left[K_{l}(n)+1, K_{r}(n)-1\right]$, so that $v_{k-1}^{n+1}, v_{k}^{n+1}, v_{k+1}^{n+1}$ are all computed from the finite-difference equation (2.2). Applying the second order difference operator $A$ to $(2.2)$ and dividing by $\Delta x^{2}$, straightforward algebra leads to

$$
\begin{aligned}
Z_{k}^{n+1}= & Z_{k}^{n}+\Delta t\left[(\mathfrak{S}+\varepsilon) \frac{A Z_{k}^{n}}{\Delta x^{2}}+2\left(\mathfrak{S}_{x}+W_{1}\right)\left(\frac{Z_{k+1}^{n}-Z_{k-1}^{n}}{2 \Delta x}\right)\right. \\
& +\mathfrak{S}_{v v}\left(W_{2}\right)^{2} \frac{Z_{k-1}^{n}+2 Z_{k}^{n}+Z_{k+1}^{n}}{4} \\
& \left.+\left\{\mathfrak{S}_{v} Z_{k}^{n} \frac{Z_{k-1}^{n}+2 Z_{k}^{n}+Z_{k+1}^{n}}{4}+2\left(\frac{Z_{k-1}^{n}+2 Z_{k}^{n}+Z_{k+1}^{n}}{4}\right)^{2}\right\}\right]
\end{aligned}
$$

with

$$
\begin{aligned}
& \mathfrak{S}:=\frac{\sigma_{k-1}^{n}+2 \sigma_{k}^{n}+\sigma_{k+1}^{n}}{4} \approx \sigma\left(v\left(x_{k}, t^{n}\right)\right), \quad \mathfrak{S}_{x}:=\frac{\sigma_{k+1}^{n}-\sigma_{k-1}^{n}}{2 \Delta x} \approx \partial_{x} \sigma\left(v\left(x_{k}, t^{n}\right)\right), \\
& \mathfrak{S}_{v}:=\frac{1}{2}\left(\frac{\sigma_{k+1}^{n}-\sigma_{k}^{n}}{v_{k+1}^{n}-v_{k}^{n}}+\frac{\sigma_{k}^{n}-\sigma_{k-1}^{n}}{v_{k}^{n}-v_{k-1}^{n}}\right) \approx \sigma^{\prime}\left(v\left(x_{k}, t^{n}\right)\right), \\
& \mathfrak{S}_{v v}:=2 \frac{\left(v_{k}^{n}-v_{k-1}^{n}\right) \sigma_{k+1}^{n}-\left(v_{k+1}^{n}-v_{k-1}^{n}\right) \sigma_{k}^{n}+\left(v_{k+1}^{n}-v_{k}^{n}\right) \sigma_{k-1}^{n}}{\left(v_{k+1}^{n}-v_{k}^{n}\right)\left(v_{k}^{n}-v_{k-1}^{n}\right)\left(v_{k+1}^{n}-v_{k-1}^{n}\right)} \approx \sigma^{\prime \prime}\left(v\left(x_{k}, t^{n}\right)\right),
\end{aligned}
$$

and

$$
W_{1}:=\frac{\bar{w}_{k-1}^{n}+2 \bar{w}_{k}^{n}+\bar{w}_{k+1}^{n}}{4} \approx \partial_{x} v\left(x_{k}, t^{n}\right), \quad W_{2}:=\bar{w}_{k}^{n} \approx \partial_{x} v\left(x_{k}, t^{n}\right)
$$

(recall that we write $\sigma_{k}^{n}=\sigma\left(v_{k}^{n}\right)$ and $\left.\bar{w}_{k}^{n}=\left(v_{k+1}^{n}-v_{k-1}^{n}\right) / 2 \Delta x\right)$. Note that (2.13) is nothing but the discrete equivalent of

$$
\partial_{t} z=\sigma(v) \partial_{x x}^{2} z+2\left[\partial_{x} \sigma(v)+\partial_{x} v\right] \partial_{x} z+\left[\sigma^{\prime \prime}(v)\left|\partial_{x} v\right|^{2}\right] z+\left[\sigma^{\prime}(v)+2\right] z^{2}
$$

for $z=\partial_{x x}^{2} v$, which is obtained differentiating twice $\partial_{t} v=\sigma(v) \partial_{x x}^{2} v+\left|\partial_{x} v\right|^{2}$ w.r.t. $x$. Let us give a formal proof that $z=\partial_{x x}^{2} v \geq \underline{z}(t)$ at the continuous level: since $0 \leq v(x, t) \leq M$ we have $0<s_{1}(M) \leq \sigma^{\prime}(v)$ and $\left|\sigma^{\prime \prime}(v)\right| \leq S_{2}(M)$, and recall that $\left|\partial_{x} v\right| \leq \gamma_{0}$. Using the definition of $\underline{z}(t)$ in Lemma 2.4 it is easy to check that $\underline{z}(t)$ is a subsolution of (2.14). Since $\underline{z}(0)=-\infty \leq z(x, 0)$ the comparison principle should give $z(x, t) \geq \underline{z}(t)$. In order to reproduce this formal computation at the discrete level let us first rewrite (2.13) as

$$
\begin{aligned}
Z_{k}^{n+1}= & {\left[1-2 \beta(\mathfrak{S}+\varepsilon)+\frac{\beta \Delta x^{2}\left(W_{2}\right)^{2} \mathfrak{S}_{v v}}{2}\right] Z_{k}^{n} } \\
& +\beta\left[(\mathfrak{S}+\varepsilon)+\frac{\Delta x^{2}\left(W_{2}\right)^{2} \mathfrak{S}_{v v}}{4}-\Delta x\left(\mathfrak{S}_{x}+W_{1}\right)\right] Z_{k-1}^{n} \\
& +\beta\left[(\mathfrak{S}+\varepsilon)+\frac{\Delta x^{2}\left(W_{2}\right)^{2} \mathfrak{S}_{v v}}{4}+\Delta x\left(\mathfrak{S}_{x}+W_{1}\right)\right] Z_{k-1}^{n} \\
& +\frac{\beta \Delta x^{2}}{8}\left(Z_{k-1}^{n}+2 Z_{k}^{n}+Z_{k+1}^{n}\right)\left[Z_{k-1}^{n}+2\left(1+\mathfrak{S}_{v}\right) Z_{k}^{n}+Z_{k+1}^{n}\right]
\end{aligned}
$$


We show now (2.15) satisfies a discrete comparison principle in the sense that $Z_{k}^{n+1}$ is non-decreasing in the three arguments $Z_{k-1}^{n}, Z_{k}^{n}, Z_{k+1}^{n}$. To this end we first note that

$$
\left|Z_{k}^{n}\right|=\left|\left(w_{k+1}^{n}-w_{k}^{n}\right) / \Delta x\right| \leq 2 \gamma_{0} / \Delta x,
$$

and by our structural hypotheses $(\mathrm{H})$ and Lemma 2.2 it is easy to check that

$$
\begin{gathered}
0 \leq \mathfrak{S} \leq \sigma(M) \quad\left|\mathfrak{S}_{x}\right| \leq S_{1}(M) \gamma_{0}, 0 \leq \mathfrak{S}_{v} \leq S_{1}(M) \\
\left|\mathfrak{S}_{v v}\right| \leq S_{2}(M), \quad\left|W_{1}\right| \leq \gamma_{0}, \quad\left|W_{2}\right| \leq \gamma_{0}
\end{gathered}
$$

Thus by the (CFL) condition

$$
\begin{aligned}
\frac{\partial Z_{k}^{n+1}}{\partial Z_{k}^{n}}= & 1-2 \beta(\mathfrak{S}+\varepsilon)+\frac{\beta \Delta x^{2}\left(W_{2}\right)^{2} \mathfrak{S}_{v v}}{2} \\
& +\frac{\beta \Delta x^{2}}{8}\left[2\left(Z_{k-1}^{n}+2\left(1+\mathfrak{S}_{v}\right) Z_{k}^{n}+Z_{k+1}^{n}\right)+2\left(1+\mathfrak{S}_{v}\right)\left(Z_{k-1}^{n}+2 Z_{k}^{n}+Z_{k+1}^{n}\right)\right] \\
\geq & 1-\beta\left[2(\sigma(M)+\varepsilon)+\frac{\Delta x^{2} \gamma_{0}^{2} S_{2}(M)}{2}+\gamma_{0} \Delta x\left(4+3 S_{1}(M)\right)\right] \geq 0, \\
\frac{\partial Z_{k}^{n+1}}{\partial Z_{k-1}^{n}}= & \beta\left[(\mathfrak{S}+\varepsilon)+\frac{\Delta x^{2}\left(W_{2}\right)^{2} \mathfrak{S}_{v v}}{4}-\Delta x\left(\mathfrak{S}_{x}+W_{1}\right)\right. \\
& \left.\left.+\frac{\Delta x^{2}}{8}\left\{\left(Z_{k-1}^{n}+2 Z_{k}^{n}+Z_{k+1}^{n}\right)+\left(Z_{k-1}^{n}+2\left(1+\mathfrak{S}_{v}\right) Z_{k}^{n}+Z_{k+1}^{n}\right)\right)\right\}\right] \\
\geq & \beta\left[\varepsilon-\gamma_{0} \Delta x\left\{\frac{\Delta x \gamma_{0} S_{2}(M)}{4}+\left(S_{1}(M)+1\right)+\frac{1}{2}\left(4+S_{1}(M)\right)\right\}\right] \geq 0,
\end{aligned}
$$

and similarly $\frac{\partial Z_{k}^{n+1}}{\partial Z_{k+1}^{n}} \geq 0$. By the induction hypothesis we see that $Z_{k}^{n+1}$ is greater or equal to the right-hand side of (2.15) evaluated with $Z_{k-1}^{n}, Z_{k}^{n}, Z_{k+1}^{n} \geq \underline{z}\left(t^{n}\right)$, and using the structural assumptions $\mathfrak{S}_{v} \geq s_{1}(M)$ and $\left|\mathfrak{S}_{v v}\right| \leq S_{2}(M)$ we get

$$
\begin{aligned}
Z_{k}^{n+1} & \geq \underline{z}\left(t^{n}\right)+\beta \Delta x^{2}\left(W_{2}\right)^{2} \mathfrak{S}_{v v} \underline{z}\left(t^{n}\right)+\beta \Delta x^{2}\left(2+\mathfrak{S}_{v}\right) \underline{z}^{2}\left(t^{n}\right) \\
& \geq \underline{z}\left(t^{n}\right)+\Delta t\left[\gamma_{0}^{2} S_{2}(M) \underline{z}\left(t^{n}\right)+\left(2+s_{1}(M)\right) \underline{z}^{2}\left(t^{n}\right)\right] .
\end{aligned}
$$

In the right-hand side we recognize $\underline{z}\left(t^{n}\right)+\Delta t F\left(\underline{z}\left(t^{n}\right)\right)$ with $F$ as in Lemma 2.4. Since by construction $\underline{\dot{z}}=F(\underline{z})$ and $\underline{z}$ is concave we finally get

$$
Z_{k}^{n+1} \geq \underline{z}\left(t^{n}\right)+\underline{\dot{z}}\left(t^{n}\right)\left[t^{n+1}-t^{n}\right] \geq \underline{z}\left(t^{n+1}\right)
$$

as required.

Step 2: Estimate close to the interfaces. We only establish the AB estimate across the right interface and boundary layer, and write again $\zeta=\zeta_{r}$ and $K=K_{r}(n)$ to keep the notations light (the argument is identical to the left). Recall that for $x_{k} \in\left[x_{K}, \zeta^{n+1}\right]$ the next step $v_{k}^{n+1} \geq 0$ is linearly interpolated by $(2.3)$, and $v_{k}^{n+1}=0$ for $x_{k} \geq \zeta^{n+1}$. As a consequence $A v_{k}^{n+1} \geq 0$ for $k>K$ and (2.12) is trivially satisfied there as $Z_{k}^{n+1} \geq 0>\underline{z}\left(t^{n+1}\right)$. Hence we only need to look at $k=K$.

By definition of $K=K_{r}(n)$ we see that $w_{K}^{n+1}, w_{K+1}^{n+1}$ satisfy (2.8) and (2.10), namely

$$
w_{K}^{n+1}=w_{K}^{n}+(a+b) \Delta x Z_{K}^{n}-(a-b) \Delta x Z_{K+1}^{n} \quad \text { and } \quad w_{K+1}^{n+1}=w_{K+1}^{n}-c \Delta x Z_{K}^{n}
$$

with $a, b$ as in (2.9) with $k=K$ and $c$ as in (2.11). Subtracting and dividing by $\Delta x$ we get that $Z_{K}^{n+1}=$ $\left(w_{K+1}^{n+1}-w_{K}^{n+1}\right) / \Delta x$ can be expressed as

$$
Z_{K}^{n+1}=(1-a-b-c) Z_{K}^{n}+(a-b) Z_{K-1}^{n} .
$$


We claim as in Step 1 that the right-hand side is nondecreasing in $Z_{K}^{n}, Z_{K-1}^{n}$. Indeed we already showed in the proof of Lemma 2.2 that $a-|b| \geq 0$, and recalling that $s^{\prime}(n)=\zeta^{n+1}-x_{K(n)} \geq \zeta^{n}-x_{K(n)} \geq \Delta x$ we compute

$$
\begin{aligned}
1-a-b-c= & 1-\beta\left(\frac{\sigma_{K}^{n}+\sigma_{K-1}^{n}}{2}+\varepsilon\right) \\
& -\beta \Delta x\left(\frac{1}{2} \frac{\sigma_{K}^{n}-\sigma_{K-1}^{n}}{v_{K}^{n}-v_{K-1}^{n}} w_{K}^{n}+\frac{w_{K-1}^{n}+2 w_{K}^{n}+w_{K+1}^{n}}{4}\right) \\
& -\frac{\beta \Delta x}{s^{\prime}(n)}\left(\left(\sigma_{K}^{n}+\varepsilon\right)-\Delta x \frac{3 w_{K+1}^{n}+w_{K}^{n}}{4}\right) \\
\geq & 1-\beta\left[(\sigma(M)+\varepsilon)+\Delta x\left(\frac{S_{1}(M) \gamma_{0}}{2}+\gamma_{0}\right)+\left(\sigma(M)+\varepsilon+\gamma_{0} \Delta x\right)\right] \geq 0,
\end{aligned}
$$

where the last inequality follows by the (CFL) condition. Before evaluating $(2.16)$ with $Z_{K}^{n}, Z_{K-1}^{n} \geq \underline{z}\left(t^{n}\right)$ we first recall from Lemma 2.3 that the interfaces propagate with discrete speed at most $\gamma_{0}$, and that by the CFL condition $\Delta t=\mathcal{O}\left(\Delta x^{2}\right)$. In particular $\Delta x \leq s(n) \leq s^{\prime}(n)=s(n)+\left(\zeta^{n+1}-\zeta^{n}\right) \leq 2 \Delta x+\mathcal{O}\left(\Delta x^{2}\right)$, thus $\Delta x \leq s^{\prime}(n) \leq 3 \Delta x$ for small $\Delta x$ (see Fig. 1) and

$$
\begin{aligned}
1-2 b-c= & 1-\beta \Delta x\left(\frac{\sigma_{K}^{n}-\sigma_{K-1}^{n}}{v_{K}^{n}-v_{K-1}^{n}} w_{K}+\frac{w_{K-1}^{n}+2 w_{K}^{n}+w_{K+1}^{n}}{2}\right) \\
& -\frac{\beta \Delta x}{\left(s^{n}\right)^{\prime}}\left(\left(\sigma_{K}^{n}+\varepsilon\right)-\Delta x \frac{3 w_{K+1}^{n}+w_{K}^{n}}{4}\right) \\
\leq & 1+\beta \gamma_{0} \Delta x\left(S_{1}(M)+2\right)-\frac{\beta \varepsilon}{3}+\beta \gamma_{0} \Delta x \\
\leq & 1-\Delta t\left(2+s_{1}(M)\right) \frac{3 \gamma_{0}}{\Delta x}
\end{aligned}
$$

by the (CFL) condition. For small $\Delta x, \Delta t$ and by definition of $t^{N}=\max \left\{t^{n^{\prime}}: F\left(t^{n^{\prime}}\right) \leq-2 \gamma_{0} / \Delta x\right\}$ it is easy to check that $F\left(t^{N}\right) \sim-2 \gamma_{0} / \Delta x$, and because $\underline{z}$ is increasing and our induction is on $n \geq N$ we can assume that $-3 \gamma_{0} / \Delta x<-2 \gamma_{0} / \Delta x \approx \underline{z}\left(t^{N}\right) \leq \underline{z}\left(t^{n}\right)$ hence

$$
1-2 b-c \leq 1+\Delta t\left(2+s_{1}(M)\right) \underline{z}\left(t^{*}\right) \leq 1+\Delta t\left(2+s_{1}(M)\right) \underline{z}\left(t^{n}\right) .
$$

Evaluating (2.16) with $Z_{k-1}^{n}, Z_{k}^{n}, Z_{k+1}^{n} \geq \underline{z}\left(t^{n}\right)$ thus gives

$$
\begin{aligned}
Z_{K}^{n+1} \geq(1-2 b-c) \underline{z}\left(t^{n}\right) & \geq \underline{z}\left(t^{n}\right)+\Delta t\left(2+s_{1}(M)\right) \underline{z}^{2}\left(t^{n}\right) \\
& \geq \underline{z}\left(t^{n}\right)+\Delta t\left[\Lambda \underline{z}\left(t^{n}\right)+\left(2+s_{1}(M)\right) \underline{z}^{2}\left(t^{n}\right)\right] \\
& =\underline{z}\left(t^{n}\right)+\Delta t F\left(\underline{z}\left(t^{n}\right)\right),
\end{aligned}
$$

and we conclude by concavity of $\underline{z}$ as in Step 1 .

Remark 2.6. For the pure PME nonlinearity $\Phi(s)=s^{m}$ one has $\sigma(r)=(m-1) r$ and therefore $s_{1}(M)=s_{1}=$ $(m-1)$ and $S_{2}(M)=0$ in $(\mathrm{H})$. The ODE for $\underline{z}$ then becomes $\dot{z}=(m+1) z^{2}$, thus $\underline{z}(t)=-1 /(m+1) t$ in Lemma 2.4 and we recover the optimal Aronson-Bénilan estimate $\partial_{x x}^{2} v \geq-1 /(m+1) t$. For general nonlinearities the optimal estimate [7] takes the form $\partial_{x x}^{2} v \geq-h(v) / t$ for some structural function $h$ related to $\Phi$. Unfortunately we were not able to reproduce the optimal computations at the discrete level, and we shall be content here with our lower bound $\partial_{x x}^{2} v \geq \underline{z}(t) \sim-C(1+1 / t)$.

Lemma 2.7. There is $C=C\left(v^{0}\right)>0$ only such that

$$
\forall n \geq 0, k \notin\left[K_{l}(n), K_{r}(n)\right]: \quad\left|\frac{v_{k}^{n+1}-v_{k}^{n}}{\Delta t}\right| \leq C .
$$


Proof. The argument is identical to ([14], Lem. 2.4).

Combining Lemmas 2.5 and 2.7 we get

Corollary 2.8. There is $C=C\left(v^{0}\right)>0$ such that

$$
\sum_{k}\left|\frac{A v_{k}^{n}}{\Delta x^{2}}\right| \Delta x+\sum_{k}\left|\frac{v_{k}^{n+1}-v_{k}^{n}}{\Delta t}\right| \Delta x \leq C\left(1+\frac{1}{t^{n}}+T\right)
$$

for all $t^{n} \leq T$.

Proof. From Lemma 2.5 and $-C(1+1 / t) \leq \underline{z}(t) \leq 0$ we see that

$$
\left|\frac{A v_{k}^{n}}{\Delta x^{2}}\right| \leq \frac{A v_{k}^{n}}{\Delta x^{2}}+2\left|\underline{z}\left(t^{n}\right)\right| \leq \frac{A v_{k}^{n}}{\Delta x^{2}}+C\left(1+\frac{1}{t^{n}}\right) .
$$

Multiplying by $\Delta x$ and summing over $k$ 's with $v_{k}^{n}=0$ outside an interval of length $C\left(1+t^{n}\right)$ (Lem. 2.3) we get the first part of the estimate

$$
\sum_{k}\left|\frac{A v_{k}^{n}}{\Delta x^{2}}\right| \Delta x \leq C\left(1+\frac{1}{t^{n}}\right)\left(1+t^{n}\right) \leq C\left(1+1 / t^{n}+T\right) .
$$

Inside the support $k \in\left[K_{l}(n), K_{r}(n)\right]$ the time derivative can be estimated from (2.2) as

$$
\begin{aligned}
\left|\frac{v_{k}^{n+1}-v_{k}^{n}}{\Delta t}\right| & =\left|\left(\sigma\left(v_{k}^{n}\right)+\varepsilon\right) \frac{A v_{k}^{n}}{\Delta x^{2}}+\left(\frac{v_{k+1}^{n}-v_{k-1}^{n}}{2 \Delta x}\right)^{2}\right| \\
& \leq(\sigma(M)+\varepsilon)\left|\frac{A v_{k}^{n}}{\Delta x^{2}}\right|+\gamma_{0}^{2} \leq C\left(\left|\frac{A v_{k}^{n}}{\Delta x^{2}}\right|+1\right),
\end{aligned}
$$

and inside the boundary layers of thickness $\Delta x \leq s(n) \leq 2 \Delta x$ the time derivative is of order $\mathcal{O}(1)$ according to Lemma 2.7. Multiplying by $\Delta x$ and summing over $k$ 's as before gives the second part of the estimate.

We end this section with uniform Höder estimates in time up to $t=0^{+}$, which are inherited from the initial Lipschitz regularity for $v^{0}(x)$.

Proposition 2.9. For any $T>0$ there is $C=C\left(T, v^{0}\right)>0$ such that

$$
\left|v_{k}^{n}-v_{k}^{m}\right| \leq C\left|t^{n}-t^{m}\right|^{1 / 2}
$$

for all $t^{n}, t^{m} \in[0, T]$.

The proof is almost identical to ([14], Lem. 2.7), and the argument is a discrete version of that in [15]. However we will need to make sure in Section 3 that the proof carries out for the hole-filling problem so we give nonetheless the full details for the sake of completeness.

Proof. We argue locally in cylinders

$$
Q=\left[x_{k_{0}}-r, x_{k_{0}}+r\right] \times\left[t^{n_{0}}, t^{n_{1}}\right],
$$

where $x_{k_{0}}$ and $0 \leq t^{n_{0}} \leq t^{n_{1}} \leq T$ are fixed and $r$ is a multiple of $\Delta x$ to be adjusted.

Step 1: Letting

$$
\begin{aligned}
H & :=\max _{n_{0} \leq n \leq n_{1}}\left|v_{k_{0}}^{n}-v_{k_{0}}^{n_{0}}\right|, \quad c:=2(\sigma(M)+\varepsilon)+\gamma_{0} r \\
V_{k}^{n} & :=v_{k}^{n}-v_{k_{0}}^{n_{0}}-\gamma_{0} r-\frac{H}{r^{2}}\left[\left(x_{k}-x_{k_{0}}\right)^{2}+c\left(t_{n}-t_{n_{0}}\right)\right],
\end{aligned}
$$


we claim that

$$
V_{k}^{n} \leq 0 \quad \text { for all }\left(x_{k}, t^{n}\right) \in Q .
$$

Arguing by induction on $n,(2.18)$ holds for $n=n_{0}$ as $V_{k}^{n_{0}} \leq\left|v_{k}^{n_{0}}-v_{k_{0}}^{n_{0}}\right|-\gamma_{0} r \leq 0$ since $\left|x_{k}-x_{k_{0}}\right| \leq r$ and $\left|w_{k}^{n}\right| \leq \gamma_{0}$. For the induction step we consider three cases: (i) $x_{k} \notin\left[\zeta_{l}^{n+1}, \zeta_{r}^{n+1}\right]$, (ii) $x_{k}$ is inside the boundary layer, and (iii) $x_{k}$ is inside the numerical support where (2.2) holds.

In the first case we have $v_{k}^{n+1}=0$ and our claim immediately holds by definition of $V_{k}^{n+1}$ with $v_{k_{0}}^{n_{0}} \geq 0$. For (ii) we have $x_{k} \in\left[x_{K(n)}, \zeta^{n+1}\right]$, and we have shown earlier that $s^{\prime}(n)=\left|\zeta^{n+1}-x_{K(n)}\right| \leq 3 \Delta x$ for small $\Delta x$. In the boundary layer $v_{k}^{n+1}$ is computed by linear interpolation with slope $\left|w_{k}^{n+1}\right| \leq \gamma_{0}$ and therefore

$$
V_{k}^{n+1} \leq v_{k}^{n+1}-\gamma_{0} \leq \gamma_{0} \cdot 3 \Delta x-\gamma_{0} r \leq 0
$$

provided that $r \geq 3 \Delta x$, which will be ensured in Step 2. In the last case (iii) we consider the linearized operator $L$ of $(2.2)$, whose action on any sequence $a_{k}^{n}$ is defined as

$$
L a_{k}^{n+1}:=\frac{a_{k}^{n+1}-a_{k}^{n}}{\Delta t}-\left(\sigma\left(v_{k}^{n}\right)+\varepsilon\right) \frac{A a_{k}^{n}}{\Delta x^{2}}-\left(\frac{v_{k+1}^{n}-v_{k-1}^{n}}{2 \Delta x}\right)\left(\frac{a_{k+1}^{n}-a_{k-1}^{n}}{2 \Delta x}\right) .
$$

Applying $L$ to $V_{k}^{n+1}$ with $L v_{k}^{n+1}=0$ as in (2.2), it is easy to compute

$$
\begin{aligned}
L V_{k}^{n+1} & =\frac{H}{r^{2}}\left[-c+2\left(\sigma\left(v_{k}^{n}\right)+\varepsilon\right)+\left(\frac{v_{k+1}^{n}-v_{k-1}^{n}}{2 \Delta x}\right)\left(x_{k}-x_{k_{0}}\right)\right] \\
& \leq \frac{H}{r^{2}}\left[-c+2(\sigma(M)+\varepsilon)+\gamma_{0} r\right] \leq 0
\end{aligned}
$$

by definition of $c$. The inequality $L V_{k}^{n+1} \leq 0$ can then be rewritten as

$$
V_{k}^{n+1} \leq(1-2 a) V_{k}^{n}+(a-b) V_{k-1}^{n}+(a+b) V_{k+1}^{n}
$$

with coefficients $a, b$ exactly as in (2.4). We already showed in the proof of Lemma 2.2 that $0 \leq a \leq 1 / 2$ and $|b| \leq a$. In particular the above right-hand side is a convex combination of $V_{k-1}^{n}, V_{k}^{n}, V_{k+1}^{n}$, thus $V_{k}^{n+1} \leq 0$ as desired.

Step 2. Choosing $k=k_{0}$ in $V_{k}^{n} \leq 0$ we see that $v_{k_{0}}^{n}-v_{k_{0}}^{n_{0}} \leq \gamma_{0} r+\frac{c H}{r^{2}}\left|t^{n_{1}}-t^{n_{0}}\right|$, and in a similar way we get the same upper bound for $v_{k_{0}}^{n_{0}}-v_{k_{0}}^{n}$. Taking the maximum over $n \in\left[n_{0}, n_{1}\right]$ and writing $s=\left|t^{n_{1}}-t^{n_{0}}\right|$ we see by definition of $H$ that

$$
H \leq \gamma_{0} r+\frac{c H}{r^{2}} s .
$$

Choose now $r$ to be a multiple of $\Delta x$ such that

$$
r_{1}+3 \Delta x \leq r \leq r_{1}+4 \Delta x
$$

where $r_{1}>0$ is the largest root of

$$
\rho^{2}-2 c s=\rho^{2}-2 \gamma_{0} s \rho+4(\sigma(M)+\varepsilon) s=0 .
$$

In particular $3 \Delta x \leq r$ as required in Step 1 , and it is easy to check that $r_{1} \lesssim C s^{1 / 2}$ when $s \leq T$. Moreover $c s / r^{2} \leq 1 / 2$ and $(2.19)$ give

$$
H / 2 \leq \gamma_{0} r \leq \gamma_{0}\left(r_{1}+4 \Delta x\right) \leq C\left(s^{1 / 2}+\Delta x\right) .
$$

Now $s=\left|t^{n_{1}}-t^{n_{0}}\right|$ and $\Delta x=\sqrt{\Delta t / \beta} \leq \beta^{-1 / 2}\left|t^{n_{1}}-t^{n_{0}}\right|^{1 / 2}$, so finally

$$
\left|v_{k_{0}}^{n_{1}}-v_{k_{0}}^{n_{0}}\right| \leq H \leq C\left|t^{n_{1}}-t^{n_{0}}\right|^{1 / 2}
$$

and the proof is complete. 


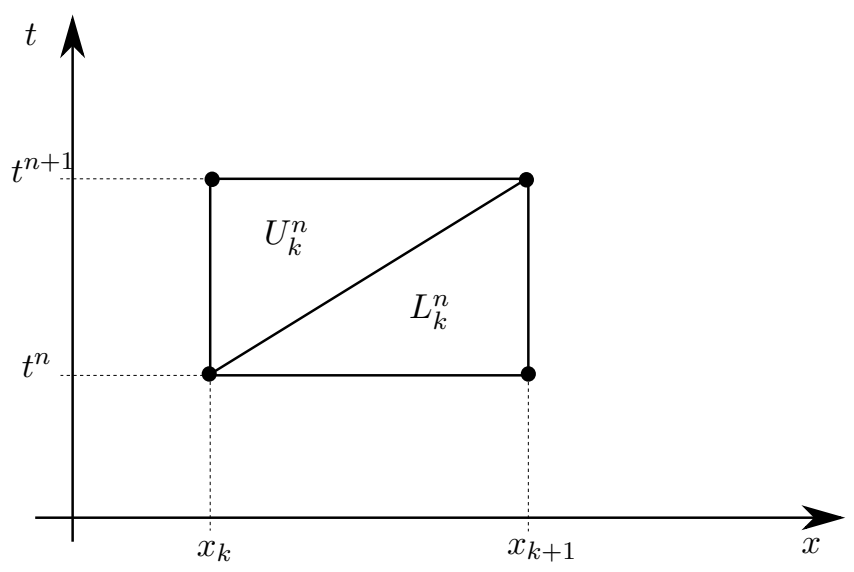

FiguRE 2. Triangular interpolation cells.

\subsection{Convergence of the approximate solution and interfaces}

Denoting $h=(\Delta t, \Delta x)$ and $L_{k}^{n}, U_{k}^{n}$ the lower and upper triangular cells in Figure 2, we first define the continuous and piecewise linear interpolation

$$
v_{h}(x, t):= \begin{cases}v_{k}^{n}+\left(x-x_{k}\right) \frac{v_{k+1}^{n}-v_{k}^{n}}{\Delta x}+\left(t-t^{n}\right) \frac{v_{k+1}^{n+1}-v_{k+1}^{n}}{\Delta t}, & (x, t) \in L_{k}^{n} \\ v_{k+1}^{n+1}+\left(x-x_{k+1}\right) \frac{v_{k+1}^{n+1}-v_{k}^{n+1}}{\Delta x}+\left(t-t^{n+1}\right) \frac{v_{k}^{n+1}-v_{k}^{n}}{\Delta t}, & (x, t) \in U_{k}^{n} .\end{cases}
$$

We also interpolate the interfaces by the piecewise linear curves

$$
\zeta_{h, l r}(t):=\zeta_{l r}^{n}+\left(t-t^{n}\right) \frac{\zeta_{l r}^{n+1}-\zeta_{l r}^{n}}{\Delta t}, \quad t \in\left[t^{n}, t^{n+1}\right] .
$$

If $Q_{T}=\mathbb{R} \times(0, T)$ the estimates from Section 2.1 can be summarized as

$$
\begin{gathered}
0 \leq v_{h}(x, t) \leq M \quad \text { and } \quad\left|\partial_{x} v_{h}(x, t)\right| \leq \gamma_{0} \quad \text { a.e. in } Q_{T}, \\
\forall t_{1}, t_{2} \in[0, T]: \quad\left|v_{h}\left(x, t_{1}\right)-v_{h}\left(x, t_{2}\right)\right| \leq C\left(T, v^{0}\right)\left|t_{1}-t_{2}\right|^{1 / 2}, \\
\forall 0<t \leq T: \quad \int_{\mathbb{R}}\left|\partial_{x x}^{2} v_{h}(., t)\right|+\int_{\mathbb{R}}\left|\partial_{t} v_{h}(., t)\right| \leq C\left(1+\frac{1}{t}+T\right)
\end{gathered}
$$

as measures in $\mathbb{R}_{x}$ (by construction $\partial_{x x}^{2} v_{h}$ consists only in Dirac masses $\delta_{x_{k}}$ ), and

$$
\left|\frac{\mathrm{d} \zeta_{h, l r}}{\mathrm{~d} t}\right| \leq \gamma_{0} \text { and } \operatorname{supp} v_{h}(., t) \subseteq\left[\zeta_{l}(0)-\Delta x-\gamma_{0} t, \zeta_{r}(0)+\gamma_{0} t+\Delta x\right] \text { for a.e. } t \in[0, T]
$$

(Lems. 2.2, 2.5 and 2.3, Prop. 2.9). The extra $\Delta x$ is needed in (2.25) because $x=\zeta^{n}$ needs not be integer meshpoints, while $v_{h}$ is interpolated from the $\left(x_{k}, t^{n}\right)$ nodes only. It is well known [8] that the Cauchy problem (1.1) has a unique solution. As in ([14], Thm. 3.3) the main convergence result then reads: 
Theorem 2.10. Let $v$ be the unique solution to (1.1) with initial datum $v^{0}$, and $\zeta_{l, r}$ the corresponding interfaces with $\operatorname{supp} v(., t)=\left[\zeta_{l}(t), \zeta_{r}(t)\right]$. Then

$$
\begin{aligned}
v_{h} \rightarrow v & \text { uniformly in } \overline{Q_{T}}, \\
\partial_{x} v_{h} \rightarrow \partial_{x} v & \text { in } L^{p}\left(Q_{T}\right) \text { for all } p \in[1, \infty), \\
\zeta_{h, l r} \rightarrow \zeta_{l r} & \text { uniformly in }[0, T]
\end{aligned}
$$

when $h=(\Delta x, \Delta t) \rightarrow 0$.

The rest of this section is devoted to the proof of Theorem 2.10, which closely follows [14].

For $(2.26)$ we show below that there is at least one subsequence $v_{h^{\prime}}$ converging to some limit $v^{*}$, and that for any such subsequence the limit $v^{*}$ is a solution to the Cauchy problem. By uniqueness $v^{*}=v$ and standard separation arguments this implies that the whole sequence $v_{h} \rightarrow v$ as in our statement.

By (2.22)-(2.23) with the upper bound (2.25) for the supports, we can extract a subsequence $\left\{h^{\prime}\right\} \subseteq\{h\}$ such that $v_{h^{\prime}} \rightarrow v^{*}$ uniformly in $\bar{Q}_{T}$ for some limit $v^{*} \in \mathcal{C}\left(\bar{Q}_{T}\right)$. For any fixed $t>0$ we see by $(2.24)$ that $\partial_{x} v_{h}(., t)$ is bounded in $B V\left(\mathbb{R}_{x}\right)$ (bounded variation) uniformly in $h^{\prime}$. By standard compactness in BV spaces [1] there is a further subsequence $\partial_{x} v_{h^{\prime \prime}}(., t) \rightarrow w^{*}$ in $L^{1}(\mathbb{R})$. By continuity we get $w^{*}()=.\partial_{x} v^{*}(., t)$, thus the whole sequence $\partial_{x} v_{h^{\prime}}(., t) \rightarrow \partial_{x} v^{*}(., t)$ for all $t>0$. An easy application of Lebesgue's dominated convergence theorem with uniform bounds $\left|\partial_{x} v_{h}\right| \leq \gamma_{0}$ gives strong $L^{p}\left(Q_{T}\right)$ convergence for all $p \in[1, \infty)$ when $h^{\prime} \rightarrow 0$ as in our statement.

We check now that the limit $v^{*}$ is indeed a solution to the Cauchy problem in the sense of Definition 1.2. Since $v_{h}^{0}(x) \rightarrow v^{0}(x)$ uniformly in $\mathbb{R}$ and $v^{*}$ is continuous up to $t=0$ the initial trace will be taken in the strong sense, and it is enough to check that

$$
\begin{aligned}
\int_{\mathbb{R}} v^{*}\left(x, t_{1}\right) \varphi\left(x, t_{1}\right) \mathrm{d} x-\int_{\mathbb{R}} v^{*}\left(x, t_{0}\right) \varphi\left(x, t_{0}\right) \mathrm{d} x \\
\quad+\int_{t_{0}} \int_{\mathbb{R}}\left\{-v^{*} \partial_{t} \varphi+\sigma\left(v^{*}\right) \partial_{x} v^{*} \partial_{x} \varphi+\left(1-\sigma^{\prime}\left(v^{*}\right)\right)\left|\partial_{x} v^{*}\right|^{2} \varphi\right\} \mathrm{d} x \mathrm{~d} t=0
\end{aligned}
$$

for all $0<t_{0} \leq t_{1} \leq T$ and test functions $\varphi \in \mathcal{C}_{c}^{\infty}\left(\bar{Q}_{T}\right)$. This weak formulation formally follows from $\partial_{t} v=$ $\sigma(v) \partial_{x x}^{2} v+\left|\partial_{x} v\right|^{2}$ after multiplying by $\varphi$ and integration by parts. Let now $\varphi_{k}^{n}:=\varphi\left(x_{k}, t^{n}\right)$, set $N_{0}:=\left\lfloor t_{0} / \Delta t\right\rfloor$ and $N_{1}:=\left\lfloor t_{1} / \Delta t\right\rfloor$, and consider the approximate Riemann sum

$$
S:=\sum_{n=N_{0}}^{N_{1}-1}\left\{\sum_{k}\left[\frac{v_{k}^{n+1}-v_{k}^{n}}{\Delta t}-\left(\sigma\left(v_{k}^{n}\right)+\varepsilon\right) \frac{A v_{k}^{n}}{\Delta x^{2}}-\left|\frac{v_{k+1}^{n}-v_{k-1}^{n}}{2 \Delta x}\right|^{2}\right] \varphi_{k}^{n} \Delta x\right\} \Delta t .
$$

By construction of our scheme the summand in $S$ is identically zero for $x_{k} \notin\left[\zeta_{l}^{n}, \zeta_{r}^{n}\right]$ and $x_{k} \in\left[x_{K_{l}(n)}, x_{K_{r}(n)}\right]$. In the remaining boundary layers, which have thickness at most $s^{n}=\left|\zeta^{n}-x_{K(n)}\right| \leq 2 \Delta x$ and where $v_{k}^{n}$ is linear, we have $\left|\left(v_{k}^{n+1}-v_{k}^{n}\right) / \Delta t\right|=\mathcal{O}(1)$ by Lemma 2.7 and $\left(\sigma\left(v_{k}^{n}\right)+\varepsilon\right) \frac{A v_{k}^{n}}{\Delta x^{2}}=\mathcal{O}(\Delta x) \frac{w_{k+1}^{n}-w_{k}^{n}}{\Delta x}=\mathcal{O}(1)$. Here we used in particular that the artificial viscosity $\varepsilon=\mathcal{O}(\Delta x)$. Thus we see that $S \rightarrow 0$ when $h^{\prime} \rightarrow 0$. Summing by parts in $S$ one can get $S=S^{\prime} \rightarrow 0$, where $S^{\prime}$ is the discrete $\Delta x \Delta t$ Riemann sum corresponding to (2.29). Using then the definition of the interpolation $v_{h^{\prime}}$ in terms of $v_{k}^{n}$, the strong convergence $v_{h^{\prime}} \rightarrow v^{*}$, the Lipschitz and Hölder regularity of $v_{h^{\prime}}, \varphi$, it is easy to express $S^{\prime}$ as the sum of dxdt integrals over all cells $L_{k}^{n}, U_{k}^{n}$, plus a remainder $o(1)$, and then send $h^{\prime} \rightarrow 0$ in order to retrieve the weak formulation $(2.29)$ for $v^{*}$ (note that $\sigma \in \mathcal{C}^{1}([0, \infty)$ ) and therefore $\sigma^{\prime}\left(v_{h}\right) \rightarrow \sigma^{\prime}(v)$ uniformly). We refer to ([14], p. 480) for the details.

Turning now to the uniform convergence of the interfaces, we only argue for the right one and write $\zeta^{n}=$ $\zeta_{r}^{n}, \zeta_{h}=\zeta_{h, r}$ and $K(n)=K_{r}(n)$ for simplicity (the proof for the left interface is exactly similar). From (2.25) 
we see that $\zeta_{h^{\prime}}$ is bounded in $W^{1, \infty}(0, T)$, so up to extraction of a further sequence if needed we may assume that $\zeta_{h^{\prime}} \rightarrow \zeta^{*}$ uniformly in $[0, T]$ for some $\zeta^{*}$. This limit $\zeta^{*}$ is moreover monotone nondecreasing in $t$ with $\zeta^{*}(0)=\zeta(0)$, as the uniform limit of the nondecreasing curves $\zeta_{h}$ with $\zeta_{h}(0)=\zeta(0)$. We shall prove that the limit agrees with the true interface $\zeta^{*}=\zeta$, and the same separation argument as before will then show that the whole sequence actually converges.

Following again [14] we first need a technical result ensuring that, at a point $\left(\zeta^{*}\left(t_{0}\right), t_{0}\right)$ where the limit curve $\zeta^{*}$ is moving with positive speed, then $v^{*}\left(., t_{0}\right)$ grows at least linearly in an interior neighborhood $\left[\zeta^{*}\left(t_{0}\right)-\delta, \zeta^{*}\left(t_{0}\right)\right]$. Again, we only state the result for the right interface and omit the subscripts.

Lemma 2.11. Let $v^{*}, \zeta^{*}=\lim v_{h^{\prime}}, \zeta_{h^{\prime}}$ as above and $\underline{z}(t)$ as in Lemma 2.4. Then

(i) For any $0<t_{0}<t_{0}+\eta \leq T$ and $\delta>0$ there holds

$$
\int_{t_{0}}^{t_{0}+\eta} v^{*}\left(\zeta^{*}(s)-\delta, s\right) \mathrm{d} s \geq \delta\left(\zeta^{*}\left(t_{0}+\eta\right)-\zeta^{*}\left(t_{0}\right)\right)-\delta^{2} \eta \underline{z}\left(t_{0}\right)
$$

(ii) If $0<t_{0}<T$ is such that $\mathrm{d} \zeta^{*} / \mathrm{d} t\left(t_{0}\right)$ exists and is positive, then there is $\delta_{0}>0$ and $c>0$ such that

$$
v^{*}\left(\zeta^{*}\left(t_{0}\right)-\delta, t_{0}\right) \geq c \delta
$$

for all $\delta \in\left[0, \delta_{0}\right]$.

This is somehow the converse statement of a well known fact for the so-called waiting-time phenomenon: if $\left(\zeta\left(t_{0}\right), t_{0}\right)$ is a free-boundary point and the pressure grows at least linearly in $x$ in an interior neighborhood $\{v>0\} \cap B_{r}\left(\zeta\left(t_{0}\right)\right) \times\left\{t_{0}\right\}$ then the free-boundary starts to move immediately, see e.g. ([22], Thm. 15.19) for a stronger statement and simple proof in dimension $d=1$ for the pure PME nonlinearity. This interpretation is purely heuristic, as we do not know at this stage that $\zeta^{*}=\lim \zeta_{h^{\prime}}$ is really the interface. Note in (ii) that $\zeta^{*} \in W^{1, \infty}(0, T)$ is differentiable a.e. $t \in(0, T)$, since $\zeta_{h}$ was uniformly bounded in $W^{1, \infty}(0, T)$.

Proof. We first give a formal proof, keeping in mind that at the discrete level we enforced $\mathrm{d} \zeta / \mathrm{d} t=-\partial_{x} v$ at the interface and that the $\mathrm{AB}$ estimate $\partial_{x x} v(x, t) \geq \underline{z}(t)$ holds. Taking $h^{\prime} \rightarrow 0$ we thus expect $\mathrm{d} \zeta^{*} / \mathrm{d} t\left(t_{0}\right)=$ $-\partial_{x} v^{*}\left(\zeta^{*}\left(t_{0}\right), t_{0}\right)$, so that $v^{*}$ should indeed grow at least linearly $\partial_{x} v\left(\zeta^{*}\left(t_{0}\right), t_{0}\right)<0$ whenever the interface is moving $\mathrm{d} \zeta^{*} / \mathrm{d} t\left(t_{0}\right)>0$. In fact (ii) rigorously follows from (i): for whenever $\zeta^{*}$ is differentiable at $t_{0}$ with $\mathrm{d} \zeta^{*} / \mathrm{d} t\left(t_{0}\right)>0$ then dividing (2.30) by $\eta \rightarrow 0$ and discarding the $\delta^{2}=o(\delta)$ term for small $\delta>0$ yields $(2.31)$ with $c \approx \mathrm{d} \zeta^{*} / \mathrm{d} t\left(t_{0}\right)>0$. Let us therefore also give a formal proof of (i): all regularity issues left aside and assuming that $v^{*}\left(\zeta^{*}(t), t\right)=0, \mathrm{~d} \zeta^{*} / \mathrm{d} t=-\partial_{x} v^{*}\left(\zeta^{*}(t), t\right)$ and $\partial_{x x}^{2} v(x, t) \geq \underline{z}(t)$ as expected, we first integrate by parts and use the generalized Aronson-Bénilan estimate to get

$$
\begin{aligned}
v^{*}\left(\zeta^{*}(s)-\delta, s\right) & =\underbrace{v^{*}\left(\zeta^{*}(s), s\right)}_{=0}-\int_{\zeta^{*}(s)-\delta}^{\zeta^{*}(s)} \partial_{x} v^{*}(x, s) \mathrm{d} x \\
& =-\int_{\zeta^{*}(s)-\delta}^{\zeta^{*}(s)}\left(\partial_{x} v^{*}\left(\zeta^{*}(s), s\right)-\int_{x}^{\zeta^{*}(s)} \partial_{x x}^{2} v^{*}(y, s) \mathrm{d} y\right) \mathrm{d} x \\
& \geq \int_{\zeta^{*}(s)-\delta}^{\zeta^{*}(s)} \underbrace{-\partial_{x} v^{*}\left(\zeta^{*}(s), s\right)}_{=+\mathrm{d} \zeta^{*} / \mathrm{d} t(s)} \mathrm{d} x+\int_{\zeta^{*}(s)-\delta}^{\zeta^{*}(s)}\left(\int_{x}^{\zeta^{*}(s)} \underline{z}(s) \mathrm{d} y\right) \mathrm{d} x \\
& \geq \delta \frac{\mathrm{d} \zeta^{*}}{\mathrm{~d} t}(s)+\int_{\zeta^{*}(s)-\delta}^{\zeta^{*}(s)} \delta \underline{z}(s) \mathrm{d} x=\delta \frac{\mathrm{d} \zeta^{*}}{\mathrm{~d} t}(s)+\delta^{2} \underline{z}(s) .
\end{aligned}
$$


Recalling that $\underline{z}(t)<0$ is monotone increasing and integrating from $t_{0}$ to $t_{0}+\eta$ we conclude that

$$
\begin{aligned}
\int_{t_{0}}^{t_{0}+\eta} v^{*}\left(\zeta^{*}(s)-\delta, s\right) \mathrm{d} s & \geq \int_{t_{0}}^{t_{0}+\eta}\left(\delta \frac{\mathrm{d} \zeta^{*}}{\mathrm{~d} t}(s)+\delta^{2} \underline{z}(s)\right) \mathrm{ds} \\
& \geq \int_{t_{0}}^{t_{0}+\eta}\left(\delta \frac{\mathrm{d} \zeta^{*}}{\mathrm{~d} t}(s)+\delta^{2} \underline{z}\left(t_{0}\right)\right) \mathrm{d} s \\
& =\delta\left(\zeta^{*}\left(t_{0}+\eta\right)-\zeta^{*}\left(t_{0}\right)\right)-\delta^{2} \eta \underline{z}\left(t_{0}\right)
\end{aligned}
$$

as desired.

Following ([14], Lem. 3.4) we now briefly sketch how to get (i) rigorously, from which (ii) will follow as already explained. For fixed $\delta, \eta, t_{0}>0$ let $p=\lfloor\delta / \Delta x\rfloor, q=\lfloor\eta / \Delta x\rfloor$, and $N=\left\lfloor t_{0} / \Delta t\right\rfloor$. Recalling that $\frac{\zeta^{n+1-\zeta^{n}}}{\Delta t}=$ $-\frac{v_{K(n)+1}^{n}-v_{K(n)}^{n}}{\Delta x}$ and summing by parts instead of integrating by parts as above, an explicit computation gives the discrete equivalent of $(2.30)$

$$
\left.\sum_{n=N}^{N+q-1} v_{K(n)-p} \Delta t \geq p \Delta x\left(\zeta^{N+q}-\zeta^{N}\right)\right)-(p \Delta x)^{2}(q \Delta t) \underline{z}\left(t^{N}\right) .
$$

Sending $h^{\prime} \rightarrow 0$ with uniform convergence $v_{h^{\prime}} \rightarrow v^{*}, \zeta_{h^{\prime}} \rightarrow \zeta^{*}$ and $x_{K(n)} \rightarrow \zeta^{*}(t)$ for $n=\lfloor t / \Delta t\rfloor$ finally allows to retrieve (2.30) and the proof is complete.

Back to the proof of $(2.28)$, we recall that we only need to establish $\lim \zeta_{h^{\prime}}=\zeta^{*}=\zeta$. From (2.25) we have $v_{h^{\prime}}(x, t)=0$ for all $x \geq \zeta_{h^{\prime}}(t)+\Delta x$. As a consequence $v^{*}(x, t)=\lim v_{h^{\prime}}(x, t)=0$ for all $x \geq \zeta^{*}(t)$, which shows by definition of $\zeta(t)=\zeta_{r}(t)=\sup \{x: v(x, t)>0\}$ that $\zeta^{*}(t) \geq \zeta(t)$. Assuming by contradiction that there is $t_{1}>0$ for which $\zeta^{*}\left(t_{1}\right)>\zeta\left(t_{1}\right)$, we claim that there is $t_{0} \in\left(0, t_{1}\right)$ such that

$$
\zeta^{*}\left(t_{0}\right)>\zeta\left(t_{0}\right) \text { and } \mathrm{d} \zeta / \mathrm{d} t\left(t_{0}\right)>0 .
$$

For if not, then arguing backwards in time starting from $t_{1}$ it is easy to see that either $\zeta^{*}(t)=$ cst. $=\zeta^{*}\left(t_{1}\right)$ for all $t \in\left[0, t_{1}\right]$, or there is $t_{2} \in\left(0, t_{1}\right)$ such that $\zeta^{*}(t)=$ cst. $=\zeta^{*}\left(t_{1}\right)$ for all $t \in\left[t_{2}, t_{1}\right]$ with $\zeta^{*}\left(t_{2}\right)=\zeta\left(t_{2}\right)$. The first case would contradict $\zeta^{*}(0)=\zeta(0)$ since $\zeta^{*}\left(t_{1}\right)>\zeta\left(t_{1}\right) \geq \zeta(0)$. In the second case, $\zeta \leq \zeta^{*}$ and the monotonicity of $\zeta$ show that necessarily $\zeta^{*}(t)=\zeta(t)=c s t=\zeta^{*}\left(t_{1}\right)$ for all $t \in\left[t_{2}, t_{1}\right]$, thus contradicting $\zeta^{*}\left(t_{1}\right)>\zeta\left(t_{1}\right)$.

For any such $t_{0}$ Lemma 2.11 gives then $v\left(\zeta^{*}\left(t_{0}\right)-\delta\right) \geq c \delta>0$ for small $\delta$ 's, and in particular choosing $0<\delta<\zeta^{*}\left(t_{0}\right)-\zeta\left(t_{0}\right)$ small enough there is a point $x_{0}=\zeta^{*}\left(t_{0}\right)-\delta>\zeta\left(t_{0}\right)$ such that $v\left(x_{0}, t_{0}\right) \geq c \delta>0$. This finally contradicts $\zeta\left(t_{0}\right)=\sup \left\{x: v\left(x, t_{0}\right)>0\right\}$ and ends the proof of Theorem 2.10.

\section{THE HOLE-FILLING PROBLEM}

In this section we choose two compactly supported "patches" $\hat{v}^{0}(x), \check{v}^{0}(x)$ such that: (i) both $\hat{v}^{0}, \check{v}^{0}$ are $\gamma_{0^{-}}$ Lipschitz, (ii) $0 \leq \hat{v}^{0}(x), \check{v}^{0}(x) \leq M$, and (iii) $\operatorname{supp} \hat{v}^{0}$ is at positive distance from supp $\check{v}^{0}$ with initial interfaces

$$
\hat{\zeta}_{l}(0)<\hat{\zeta}_{r}(0)<\check{\zeta}_{l}(0)<\check{\zeta}_{r}(0) \text {. }
$$

Defining

$$
v^{0}:=\max \left\{\hat{v}^{0}, \check{v}^{0}\right\}
$$

this means that $\operatorname{supp} v^{0}=\operatorname{supp} \hat{v}^{0} \cup \operatorname{supp} \check{v}^{0}$ has an internal hole of width $d_{0}=\check{\zeta}_{l}(0)-\hat{\zeta}_{r}(0)>0$ between $\operatorname{supp} \hat{v}^{0}$ to the left and $\operatorname{supp} \check{v}^{0}$ to the right. Let $v(x, t), \hat{v}(x, t)$, and $\check{v}(x, t)$ be the solution of the Cauchy problem with initial data respectively $v^{0}(x), \hat{v}^{0}(x)$, and $\check{v}^{0}(x)$. We are interested here in computing a numerical approximation to $v(x, t)$. By noncontraction of the supports we know that $\hat{\zeta}_{r}(t)$ is nondecreasing, $\check{\zeta}_{l}(t)$ is nonincreasing, and because the interfaces propagate with finite speed at most $\gamma_{0}$ (which also follows from Sect. 2) the first time when the supports touch

$$
0<T^{*}=\sup \left\{t \geq 0: \quad \hat{\zeta}_{r}(t)<\check{\zeta}_{l}(t)\right\} \leq \infty
$$


is positive. By uniqueness this implies that

$$
v=\max \{\hat{v}, \check{v}\} \quad \text { in }\left[0, T^{*}\right),
$$

so for $t \in\left[0, T^{*}\right)$ an internal hole of width $\mathrm{d}(t)=\check{\zeta}_{l}(t)-\hat{\zeta}_{r}(t)>0$ persists in the support of $v$ between the left and right patches $\hat{v}, \check{v}$. A well-known property of GPME is that "once an interface starts moving it never stops", see e.g. ([22], Lem. 14.20) in any dimension for the pure PME nonlinearity and ([22], Cor. 15.23) for a simple proof in dimension one. Since the internal interfaces were at positive distance at time 0 this implies that, if and when they meet in finite time $\hat{\zeta}_{r}\left(T^{*}\right)=x^{*}=\check{\zeta}_{l}\left(T^{*}\right)$, at least one of the internal interfaces has started moving (otherwise the two would not meet) and is therefore still moving with positive speed. As a consequence at least one of the patches $\hat{v}, \check{v}$ becomes instantaneously positive at $x=x^{*}$ for $t>T^{*}$, the comparison principle then implies $v\left(x^{*}, t\right) \geq \max \left\{\hat{v}\left(x^{*}, t\right), \breve{v}\left(x^{*}, t\right)\right\}>0$, and the hole eventually disappears at $t=T^{*}$. Once the hole has filled the internal interfaces disappear, $\operatorname{supp} v(., t)$ becomes a connected interval $\left[\zeta_{l}(t), \zeta_{r}(t)\right]$ (containing the whole $\left.\left[\hat{\zeta}_{l}\left(T^{*}\right), \check{\zeta}_{r}\left(T^{*}\right)\right]\right)$, and $v$ does not equal $\max \{\hat{v}, \check{v}\}$ anymore.

In Section 2 we described how to compute the approximate solution and interfaces when the initial datum consists in a single patch, which is exactly our assumption for both $\hat{v}^{0}, \hat{v}^{0}$. Using the results in the previous section we can therefore construct an approximation to each of the corresponding solutions $\hat{v}, \check{v}$ and track all the resulting interfaces. We explain below how this previous one-patch algorithm can be naturally extended to the above case of two initial patches, while tracking all the interfaces (internal and external), detecting the hole-filling with accuracy, and solving past this time.

Remark 3.1. We discuss here the case of two patches only for the ease of exposition, but the argument is easily adapted to any arbitrary number of initial patches at positive distance one from each other.

Roughly speaking, the algorithm goes as follows: starting from $\hat{v}_{k}^{0}, \check{v}_{k}^{0}$, construct two independent sets of approximate solutions and interfaces $\left(\hat{v}_{k}^{n}, \hat{\zeta}_{l, r}^{n}\right)$ and $\left(\hat{v}_{k}^{n}, \hat{\zeta}_{l, r}^{n}\right)$ applying the one-patch scheme from Section 2 separately to each patch. As long as the internal interfaces $\hat{\zeta}_{r}, \check{\zeta}_{l}$ do not meet keep solving, and define $v_{k}^{n}=$ $\max \left\{\hat{v}_{k}^{n}, \breve{v}_{k}^{n}\right\}$. If the internal interfaces meet at $t=t^{N}$ then stop tracking them, define the external interfaces $\zeta_{l}^{N}:=\hat{\zeta}_{l}^{N}, \zeta_{r}^{N}:=\check{\zeta}_{r}^{N}$, and resume the computation applying the one-patch scheme to $v_{k}^{n}$ starting from $v_{k}^{N}$ at time $t^{N}$. More precisely,

Algorithm 1 (Numerical scheme for the hole-filling). Initialize $\hat{v}_{k}^{0}:=\hat{v}^{0}\left(x_{k}\right), \check{v}_{k}^{0}:=\check{v}^{0}\left(x_{k}\right), v_{k}^{0}:=\max \left\{\hat{v}_{k}^{0}, \check{v}_{k}^{0}\right\}$, as well as $\hat{\zeta}_{l, r}^{0}:=\hat{\zeta}_{l, r}(0), \check{\zeta}_{l, r}^{0}:=\check{\zeta}_{l, r}(0)$, and $\zeta_{l}^{0}:=\hat{\zeta}_{l}^{0}, \zeta_{r}^{0}:=\check{\zeta}_{r}^{0}$. For fixed $T>0$ and while $t^{n} \leq T$, do:

1. Apply the one-patch algorithm from Section 2 separately to $\hat{v}^{n}, \hat{\zeta}_{l, r}^{n}$ and $\check{v}^{n}, \check{\zeta}_{l, r}^{n}$ in order to compute a temporary prediction $\hat{v}^{(n+1)^{\prime}}, \hat{\zeta}_{l, r}^{(n+1)^{\prime}}$ and $\check{v}^{(n+1)^{\prime}}, \check{\zeta}_{l, r}^{(n+1)^{\prime}}$. If the predicted internal interfaces are at least $\Delta x$ away $\check{\zeta}_{l}^{(n+1)^{\prime}}-\hat{\zeta}_{r}^{(n+1)^{\prime}}>\Delta x$, update all quantities $(n+1)^{\prime} \rightarrow(n+1)$, set $v_{k}^{n+1}:=\max \left\{\hat{v}_{k}^{n+1}, \check{v}_{k}^{n+1}\right\}$, and repeat Step 1. Otherwise mark the numerical filling time $T_{h}^{*}:=t^{n}$, redefine the external interfaces $\zeta_{l}^{n}:=\hat{\zeta}_{l}^{n}$ and $\zeta_{r}^{n}:=\check{\zeta}_{r}^{n}$, and go to Step 2.

2. Apply the one-patch algorithm from Section 2 to $v^{n}, \zeta_{l, r}^{n}$ in order to construct $v^{n+1}, \zeta_{l, r}^{n+1}$, and repeat Step 2.

Note that, because the internal interfaces propagate with discrete speed at most $\gamma_{0}$ (Lem. 2.3) and start at an initial distance $d(0)>0$, Step 1 will be applied at least for $t^{n} \leq d(0) / 2 \gamma_{0}$ hence $T_{h}^{*} \geq d(0) / 2 \gamma_{0}$ uniformly in $h$. In case the filling does occur for some $T_{h}^{*} \leq T$ then the numerical internal interfaces are not defined for later times.

\subsection{A priori estimates}

We show here that all the previous discrete estimates are preserved across and after the filling time, including the $L^{\infty}$, Lipschitz, and Hölder bounds as well as the generalized Aronson-Bénilan estimate. In particular we will obtain that the pressure $v$ stays $\gamma_{0}$-Lipshitz after the filling time, which only holds in dimension one because 
$w=\partial_{x} v$ satisfies a maximum principle (see the proof of Lem. 2.2). In higher dimensions there is no such maximum principle, and the global Lipschitz regularity of the pressure is known to fail precisely because of the hole-filling phenomenon [11,22] ( $|\nabla v|$ blows up where and when internal holes fill). As in the previous section and without further mention we impose the (CFL) condition on the mesh parameters $\Delta x, \Delta t, \varepsilon$.

Proposition 3.2. Let $v_{k}^{n}$ be the (two-patches) discrete solution constructed with Algorithm 1 , and $\underline{z}(t)<0$ as in Lemma 2.4. Then

$$
0 \leq v_{k}^{n} \leq M, \quad\left|\frac{v_{k}^{n}-v_{k-1}^{n}}{\Delta x}\right| \leq \gamma_{0}, \quad\left|v_{k}^{n}-v_{k}^{m}\right| \leq C\left|t^{n}-t^{m}\right|^{1 / 2}, \quad \frac{A v_{k}^{n}}{\Delta x^{2}} \geq \underline{z}\left(t^{n}\right)
$$

hold for all $k$ and $t^{n}, t^{m} \in[0, T]$.

Proof. If no (numerical) hole filling occurs our statement immediately follows from the results in Section 2, as $v_{k}^{n}$ coincides with either $\hat{v}_{k}^{n}$ or $\breve{v}_{k}^{n}$ on each side of the internal hole of width at least $\Delta x$. Thus we may assume that the internal interfaces meet at $t=t^{N}$.

For times $t^{n} \leq t^{N}$ the patches $0 \leq \hat{v}_{k}^{n}, \check{v}_{k}^{n} \leq M$ are $\gamma_{0}$-Lipschitz (Lem. 2.2) so clearly $v_{k}^{n}=\max \left\{\hat{v}_{k}^{n}, \check{v}_{k}^{n}\right\}$ satisfies the same bounds for all $t^{n} \leq t^{N}$, and in particular at $t=t^{N}$. By definition $v_{k}^{n}$ is then constructed for $t^{n} \geq t^{N}$ by applying the one-patch scheme to solve the discrete Cauchy problem starting from the initial data $v_{k}^{N}$ at time $t^{N}$. Since $v_{k}^{N}$ satisfies the desired bounds at $t=t^{N}$ we conclude by Lemma 2.2 that $v_{k}^{n}$ satisfies the same $L^{\infty}$ and $\gamma_{0}$-Lipschitz estimates for all $t^{n} \geq t^{N}$.

Regarding now the Hölder continuity in time, we check that the proof of Proposition 2.9 still applies. In Step 1 $\left(V_{k}^{n} \leq 0\right.$ in $Q$ by induction on $\left.n \in\left[n_{0}, n_{1}\right]\right)$ the initialization $n=n_{0}$ only requires $\gamma_{0}$-Lipschitz bounds, which is true here. For the induction step we distinguished three cases: (i) $x_{k}$ is outside of the support with $v_{k}^{n}=0$, (ii) $x_{k}$ is within one of the boundary layers, and (iii) when $v_{k}^{n+1}$ is constructed applying the finite-difference scheme (2.2). All three cases are easily checked here with two patches: (i) and (ii) are identical, and (iii) also works here since $v_{k}^{n+1}$ is in fact constructed applying the finite-difference equation (2.2) to either one of the two patches before the filling time and to the unique patch afterward. Step 2 is identical, since it relies only on structural considerations and the previous $L^{\infty}$ and Lipschitz bounds.

We finally turn to the $\mathrm{AB}$ estimate. By definition of the hitting time $t^{N}$ we have that $\check{\zeta}_{l}^{n}-\hat{\zeta}_{r}^{n}>\Delta x$ stay strictly $\Delta x$ away from each other for $t^{n} \leq t^{N}$, so that there is always at least one integer mesh point in the hole. Since $v_{k}^{n} \geq 0$ everywhere and $v_{k}^{n}=0$ in the hole it is easy to check that $A v_{k}^{n} \geq 0$ for all $x_{k}$ such that $\hat{\zeta}_{r}^{n}-\Delta x \leq x_{k} \leq \check{\zeta}_{l}^{n}+\Delta x$, hence the AB estimate is trivially satisfied there (recall that $\underline{z}(t)<0$ ). Now outside the hole $A v_{k}^{n}$ equals either $A \hat{v}_{k}^{n}$ or $A \check{v}_{k}^{n}$, hence the $\mathrm{AB}$ estimate holds for all $t^{n} \leq t^{N}$ and including at $t=t^{N}$. Now for $t^{n} \geq t^{N}$ the solution $v_{k}^{n}$ is constructed applying the one-patch algorithm with initial datum $v_{k}^{N}$ at time $t^{N}$, which satisfies the $\mathrm{AB}$ estimate. By Lemma 2.5 we conclude that the estimate also holds for all $t^{n} \geq t^{N}$ and the proof is complete.

\subsection{Convergence of the approximate solutions and interfaces}

For fixed $T>0$ we denote $Q_{T}=\mathbb{R} \times(0, T)$ and $h=(\Delta x, \Delta t)$ as before. As in Section 2.2 we define $v_{h}$ to be continuous and piecewise linear in all triangle $L_{k}^{n}, U_{k}^{n}$ according to (2.20). The external $\zeta_{h, l r}$ and internal $\hat{\zeta}_{h, r}, \check{\zeta}_{h, l}$ interfaces are defined to be piecewise linear as in (2.21). Note that $\zeta_{h, l r}$ are defined up to $t=T$, while $\hat{\zeta}_{h, r} \leq \check{\zeta}_{h, l}$ are only defined up to the (numerical) filling time

$$
T_{h}^{*}:=\max \left\{t^{n}: \quad \check{\zeta}_{l}^{n}-\hat{\zeta}_{r}^{n}>\Delta x\right\}
$$

(see Algorithm 1). If no filling is numerically detected before the end of the computation we simply do not define $T_{h}^{*}$. In any case the internal interfaces $\hat{\zeta}_{h, r}, \check{\zeta}_{h, l}$ are respectively monotone nondecreasing and nonincreasing as long as they exist.

Theorem 3.3. For fixed $T>0$ the numerical solution $v_{h}$ converges uniformly in $\bar{Q}_{T}$ to the unique solution $v$ when $h \rightarrow 0$. 
Proof. Note that the proof of (2.26) for the case of a single patch in Theorem 2.10 only relies on: (i) the discrete estimates on $v_{h}$ uniformly in $h$ allowing to get strong compactness for $v_{h}$ and $\partial_{x} v_{h}$; (ii) uniqueness for the Cauchy problem; (iii) the consistence of the finite-difference equation (2.2) inside the support; and (iv) the fact that all quantities involved in $(2.2)$ are of order $\mathcal{O}(1)$ inside the numerical boundary layers, see Section 2.2 for the details. By Proposition 3.2 this remains true in the case of two patches, thus allowing to conclude exactly as in the proof of Theorem 2.10.

The uniform convergence of the interfaces is now more delicate, as we need to distinguish between cases depending on whether the hole fills or not before the computation time $T$. Roughly speaking, as long as the interfaces make sense the convergence follows as in the case of one patch only. We prove in particular that, if and when the numerical filling occurs at time $t=T_{h}^{*}$, then $T_{h}^{*}$ is indeed a good approximation to the exact filling time $T^{*}$ :

Theorem 3.4. Fix $T>0$ and let $T^{*}$ be the theoretical hole-filling time.

(a) If $T^{*}<T$ then there is a small $\delta_{0}>0$ such that the numerical filling eventually occurs at times $T_{h}^{*} \leq T-\delta_{0}$ for all $h \leq h_{0}$, and $\lim _{h \rightarrow 0} T_{h}^{*}=T^{*}$. Moreover

$$
\left\|\zeta_{h, l}-\zeta_{l}\right\|_{L^{\infty}(0, T)}+\left\|\zeta_{h, r}-\zeta_{r}\right\|_{L^{\infty}(0, T)} \rightarrow 0
$$

and

$$
\left\|\hat{\zeta}_{h, r}-\hat{\zeta}_{r}\right\|_{L^{\infty}\left(0, T^{*}-\eta\right)}+\left\|\check{\zeta}_{h, l}-\check{\zeta}_{l}\right\|_{L^{\infty}\left(0, T^{*}-\eta\right)} \rightarrow 0
$$

when $h \rightarrow 0$ for any small $\eta>0$ fixed.

(b) If $T^{*} \geq T$ then for all $\eta>0$ there exists $h_{0}(\eta)$ such that for all $h \leq h_{0}$ either no numerical filling occurs before $t=T$, or does so at times $T_{h}^{*} \geq T-\eta$. In particular for small $\eta$ the internal interfaces $\hat{\zeta}_{h, r}, \check{\zeta}_{h, l}$ are defined at least for $t \leq T-\eta$. Moreover

$$
\left\|\zeta_{h, l}-\zeta_{l}\right\|_{L^{\infty}(0, T)}+\left\|\zeta_{h, r}-\zeta_{r}\right\|_{L^{\infty}(0, T)} \rightarrow 0
$$

and

$$
\left\|\hat{\zeta}_{h, r}-\hat{\zeta}_{r}\right\|_{L^{\infty}(0, T-\eta)}+\left\|\check{\zeta}_{h, l}-\check{\zeta}_{l}\right\|_{L^{\infty}(0, T-\eta)} \rightarrow 0
$$

when $h \rightarrow 0$ for any small $\eta>0$ fixed.

Practically speaking this means that if a hole-filling is detected numerically at $t=T_{h}^{*}$ then indeed $T_{h}^{*}$ is a good approximation to the theoretical filling time $T^{*}$, while if no hole-filling is detected before the end of the computation then one has simply not waited long enough to see the hole-filling, i.e. $T^{*} \geq T$. In any case the numerical interfaces converge to the theoretical ones, both internal (as long as they exist) and external (up to $t=T)$

Before going into the details, it is worth pointing out that at the filling time there holds

$$
0 \leq \check{\zeta}_{h, l}\left(T_{h}^{*}\right)-\hat{\zeta}_{h, r}\left(T_{h}^{*}\right) \leq \mathcal{O}(\Delta x) .
$$

Indeed by (3.1) we have $T_{h}^{*}=t^{N}$ for some $N$, which according to Algorithm 1 is characterized by the fact that $\check{\zeta}_{l}^{N}-\hat{\zeta}_{r}^{N}>\Delta x$ and virtually computing one more step separately for each patch would result in $\check{\zeta}_{l}^{N+1}-\hat{\zeta}_{r}^{N+1} \leq$ $\Delta x$. Recalling that any interface propagates with discrete speed at most $\gamma_{0}$ (Lem. 2.3) we see that indeed $0 \leq \check{\zeta}_{l}^{N}-\hat{\zeta}_{r}^{N} \leq\left(\check{\zeta}_{l}^{N+1}-\hat{\zeta}_{r}^{N+1}\right)+2 \gamma_{0} \Delta t \leq \Delta x+2 \gamma_{0} \Delta t \leq \mathcal{O}(\Delta x)$ since $\Delta t=\mathcal{O}\left(\Delta x^{2}\right)$.

Proof of (a). We first show that the hole-filling always eventually occurs before the end of the computation if $h$ is small enough, i.e. $T_{h}^{*} \leq T-\delta_{0}$ as in our statement. Assuming by contradiction that this does no hold, then by definition of $T_{h}^{*}$ there is a discrete subsequence (not relabeled) such that either no numerical filling occurs before $t=T$, or does so for times $T_{h}^{*} \nearrow T$. In any case and by definition of the internal interfaces we can find a sequence of points $\left(x_{h}, t_{h}\right)$ such that $t_{h} \nearrow T$ and $x_{h} \in\left[\hat{\zeta}_{h, r}\left(t_{h}\right), \check{\zeta}_{h, l}\left(t_{h}\right)\right]$ with $v_{h}\left(x_{h}, t_{h}\right)=0$. 
By monotonicity of the interfaces we see that $x_{h}$ stays in the fixed compact set $\left[\hat{\zeta}_{r}(0), \check{\zeta}_{l}(0)\right]$, so up to extracting a further subsequence we can assume that $x_{h} \rightarrow x_{0} \in\left[\hat{\zeta}_{r}(0), \check{\zeta}_{l}(0)\right]$. By Theorem 3.3 we get

$$
v\left(x_{0}, T\right)=\lim _{h \searrow 0} v_{h}\left(x_{h}, T_{h}^{*}\right)=0 \quad \text { for some } x_{0} \in\left[\hat{\zeta}_{r}(0), \check{\zeta}_{l}(0)\right] .
$$

We argue now for the theoretical solution and interfaces in order to get a contradiction. Because $T^{*}<T$ and the internal interfaces start at positive distance from each other they must meet at $x^{*}=\hat{\zeta}_{r}\left(T^{*}\right)=\check{\zeta}_{l}\left(T^{*}\right) \in$ $\left[\hat{\zeta}_{r}(0), \check{\zeta}_{l}(0)\right]$, and necessarily one of them has started moving before $t=T^{*}$ (otherwise they would not meet). Once an interface starts moving it never stops, so at least one of the interfaces is really moving at $t=T^{*}$ and thus $\hat{v}\left(x^{*}, t\right)>0$ or $\check{v}\left(x^{*}, t\right)>0$ for all $t>T^{*}$. By the comparison principle $v \geq \max \{\hat{v}, \breve{v}\}$ is positive everywhere in $\left[\hat{\zeta}_{r}(0), \breve{\zeta}_{l}(0)\right]$ for all $t>T^{*}$, in particular for $t=T>T^{*}$. This finally contradicts $v\left(x_{0}, T\right)=0$.

We claim now that $\lim _{h \backslash 0} T_{h}^{*}=T^{*}$. Since $0 \leq T_{h}^{*} \leq T-\delta_{0}$ for small $h$, we can extract a subsequence such that $T_{h^{\prime}}^{*} \rightarrow \tilde{T}^{*}$ for some $\tilde{T}^{*}<T$. We prove that necessarily $\tilde{T}^{*}=T^{*}$, which will show that the whole sequence converges. Virtually keeping applying the one-patch algorithm separately to each of the patches $\hat{v}_{h^{\prime}} \check{v}_{h^{\prime}}$ after $t=T_{h^{\prime}}^{*}$, we can naturally extend $\hat{\zeta}_{h^{\prime}, r}, \check{\zeta}_{h^{\prime}, l}$ to all $t \in[0, T]$. By construction of our scheme these (virtually) extended interfaces, still denoted $\hat{\zeta}_{h^{\prime}, r}, \check{\zeta}_{h^{\prime}, l}$ with a slight abuse of notations, coincide with the internal interfaces for $v_{h^{\prime}}$ up to the numerical filling time $T_{h}^{*}$, after which we stop tracking the internal interfaces but the extended ones virtually still exist up to $t=T$. Applying Theorem 2.10 we see that the extended interfaces $\hat{\zeta}_{h^{\prime}, r}, \check{\zeta}_{h^{\prime}, l} \rightarrow$ $h a t \zeta_{r}, \check{\zeta}_{l}$ uniformly in $[0, T]$, where $\hat{\zeta}, \check{\zeta}$ are the interfaces of each patch $\hat{v}, \check{v}$ considered as two independent solutions up to $t=T$. Since $T_{h^{\prime}}^{*} \rightarrow \tilde{T}^{*}$ we get by (3.2) and uniform convergence that

$$
\hat{\zeta}_{r}\left(\tilde{T}^{*}\right)-\check{\zeta}_{l}\left(\tilde{T}^{*}\right)=\lim _{h^{\prime} \rightarrow 0}\left(\hat{\zeta}_{h^{\prime}, r}\left(T_{h^{\prime}}^{*}\right)-\check{\zeta}_{h^{\prime}, l}\left(T_{h^{\prime}}^{*}\right)\right)=0 .
$$

Because $\hat{\zeta}_{r}, \check{\zeta}_{l}$ are monotone and start at positive distance and once an interface starts moving it never stops, they can only meet at a unique time. By definition this time is $t=T^{*}$, thus $\tilde{T}^{*}=T^{*}$ and $T_{h}^{*} \rightarrow T^{*}$ as desired.

Uniform convergence of the interfaces can be obtained as in the proof of Theorem 2.10 as long as the internal interfaces exist and are tracked numerically (this is why we need to step $\eta>0$ away from $T^{*}$ as in our statement, thus ensuring that the internal interfaces are numerically defined at least for fixed time intervals $\left[0, T^{*}-\eta\right]$ ), and the proof is achieved.

Proof of (b). We claim that a hole-filling can only be detected numerically for times $T_{h}^{*} \geq T-\eta$ close to the total computation time $T$ if $h$ is small enough (and may actually not be detected). For if not, then $T_{h^{\prime}}^{*} \leq T-\delta_{0}$ for some subsequence and fixed $\delta_{0}>0$. Arguing exactly as in (a) we conclude that $T_{h^{\prime}}^{*} \rightarrow T^{*}$, which shows in particular that $T^{*} \leq T-\delta_{0}$ and contradicts $T^{*} \geq T$. The convergence of the interfaces is also exactly similar to the proof of Theorem 2.10, stepping again $\eta>0$ away from $t=T$ for the internal interfaces as in our statement.

\section{Numerical EXPERIMENTS}

The stability (CFL) condition was imposed in order to ensure Lipschitz bounds and $L^{\infty}$ stability of the scheme (Lem. 2.2), but also the generalized Aronson-Bénilan estimate (Lem. 2.5). For numerical purposes the less stringent condition

$$
\beta \leq \frac{1}{2(\sigma(M)+\varepsilon)} \quad \text { and } \quad \gamma_{0} \Delta x\left(1+S_{1}(M) / 2\right) \leq \varepsilon \leq \mathcal{O}(\Delta x)
$$

suffices to guarantee the stability Lemma 2.2 and seems to give satisfactory convergence (see below). Note that in contrast with (CFL) this relaxed condition does not depend on $s_{1}(M), S_{2}(M)$ anymore. In any case the computationally expensive $\beta=\Delta t / \Delta x^{2}=\mathcal{O}(1)$ condition is necessary due to the explicit nature of the scheme. In [17] Hoff considered a linearly implicit version of [14] for the pure PME nonlinearity. We presented here the 

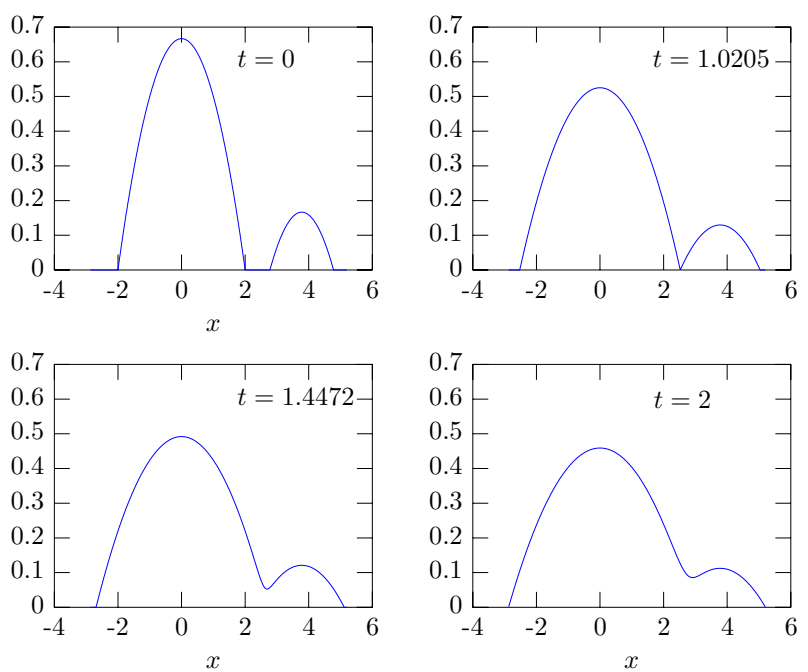

FiguRE 3. Numerical solution $v_{h}(., t)$ for several times $(\Delta x=0.01)$.

explicit scheme for the ease of exposition, but all the theoretical results in Sections 2 and 3 extend to general nonlinearities by considering as in [17] the linearly implicit finite-difference equation

$$
\frac{v_{k}^{n+1}-v_{k}^{n}}{\Delta t}=\sigma\left(v_{k}^{n}\right) \frac{A v_{k}^{n+1}}{\Delta x^{2}}+\varepsilon \frac{A v_{k}^{n}}{\Delta x^{2}}+\left|\frac{v_{k+1}^{n}-v_{k-1}^{n}}{2 \Delta x}\right|^{2}
$$

instead of (2.2). In this case the stability condition becomes $\Delta t=\mathcal{O}(\Delta x)$, which is clearly the best one can hope for since the propagation law $\mathrm{d} \zeta / \mathrm{d} t=-\partial_{x} v$ is intrinsically hyperbolic.

In order to test our scheme and because no explicit solutions are known for general nonlinearities we restrict to the pure PME $\partial_{t} v=(m-1) v \partial_{x x}^{2} v+\left|\partial_{x} v\right|^{2}$, to which the Barenblatt profiles

$$
t \geq-t_{0}: \quad V_{m}\left(x, t ; C, x_{0}, t_{0}\right)=\frac{1}{t_{0}+t}\left(C\left(t_{0}+t\right)^{2 /(m+1)}-\frac{1}{2(m+1)}\left|x-x_{0}\right|^{2}\right)_{+}
$$

are exact solutions for any $m>1$. Here $C>0$ is a free parameter, while $x_{0}, t_{0}$ reflect the invariance under shifts. The interfaces are then explicitly given by

$$
\zeta_{l r}(t)=x_{0} \pm \sqrt{2(m+1) C}\left(t_{0}+t\right)^{1 /(m+1)} .
$$

For our numerical experiment we fix $m=2$ and choose arbitrary parameters

$$
\hat{v}(x, t):=V_{2}(x, t ; 4 / 6,0,1), \quad \check{v}(x, t):=V_{2}(x, t ; 1 / 6,3 \sqrt[3]{2}, 1)
$$

for which the initial supports of $\hat{v}^{0}(x):=\hat{v}(x, 0), \check{v}^{0}(x):=\check{v}(x, 0)$ are at positive distance from each other as in Section 3, and the corresponding interfaces are then

$$
\hat{\zeta}_{l r}(r)=0 \pm 2(1+t)^{1 / 3}, \quad \check{\zeta}_{l r}(t)=3 \sqrt[3]{2} \pm(1+t)^{1 / 3} .
$$

Starting with initial datum $v_{0}=\max \left\{\hat{v}^{0}, \check{v}^{0}\right\}$ the theoretical hole-filling time $T^{*}$ can be computed according to Section 3 by solving $\hat{\zeta}_{r}(t)=\check{\zeta}_{l}(t) \Leftrightarrow t=T^{*}$, which with our specific parameters gives explicitly

$$
T^{*}=1, \quad x^{*}=\hat{\zeta}_{r}\left(T^{*}\right)=\check{\zeta}_{l}\left(T^{*}\right)=2 \sqrt[3]{2} \approx 2.5198 .
$$

All the computations were performed on a personal computer with Linux/Octave. We only specify the value of $\Delta x$, the parameters $\Delta t, \varepsilon$ being then chosen respectively with the largest and smallest value allowed by (CFL'). Figure 3 shows a typical solution $v_{h}(x, t)$ with $\Delta x=0.01$, and Figure 4 illustrates the corresponding numerical 


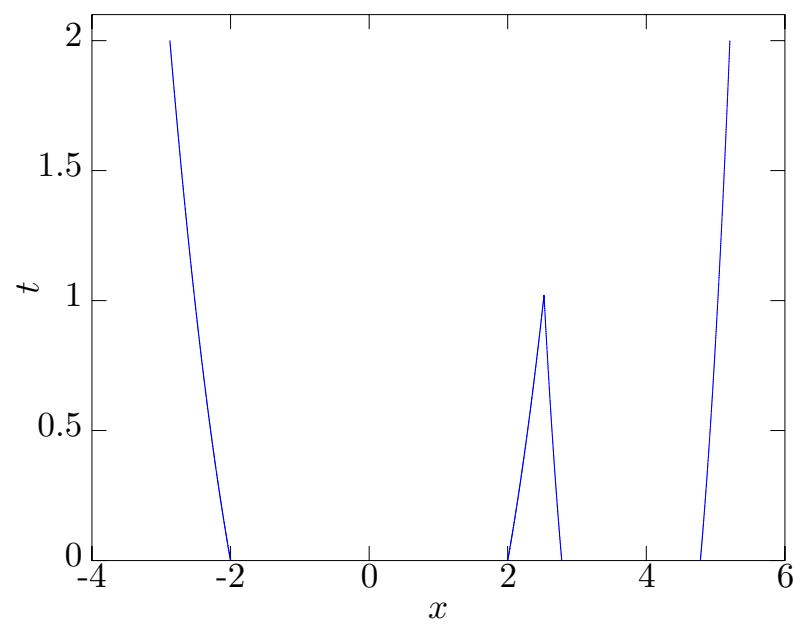

FigURE 4. Interface curves $(\Delta x=0.01)$.
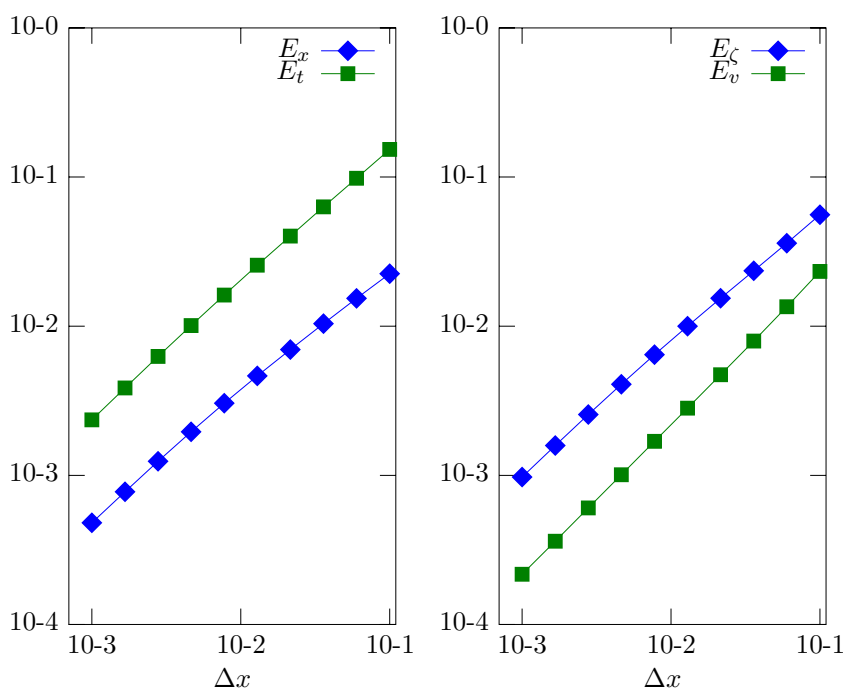

Figure 5. Errors as a function of $\Delta x$.

interfaces. The hole filling was numerically detected for $T_{h}^{*}=1.0205$ and $x_{h}^{*}=2.5236\left(\right.$ compare with $T^{*}=1$ and $x^{*}=2 \sqrt[3]{2} \approx 2.5198$ ).

In addition to an abstract convergence result as in Theorem 2.10 DiBenedetto and Hoff also derived explicit error estimates for the pure PME nonlinearity in the case of one single patch, in the form $\left\|\zeta_{h}-\zeta\right\|_{L^{\infty}(0, T)}+$ $\left\|v_{h}-v\right\|_{L^{\infty}\left(Q_{T}\right)} \leq \mathcal{O}\left(\Delta x^{\alpha}|\log \Delta x|^{\beta}\right)$ for some structural $\alpha, \beta$ related to $m>1$, see ([14], Thm. 4.1). However their proof heavily relies on the explicit power structure $\Phi(s)=s^{m}$, and obtaining error estimates for general nonlinearities is a hard task that we did not carry out here due to the technical difficulties and lack of space. Figure 5 shows the numerical errors $E_{x}:=\left|x_{h}^{*}-x^{*}\right|, E_{t}=\left|T_{h}^{*}-T^{*}\right|$ and $E_{\zeta}=\left\|\zeta_{h}-\zeta\right\|_{\left.L^{\infty}\left(0, T_{h}^{*}\right)\right)}, E_{v}=$ $\left\|v_{h}-v\right\|_{L^{\infty}\left(Q_{T_{h}^{*}}\right)}$ as a function of $\Delta x$, and strongly suggests $\mathcal{O}\left(\Delta x^{\alpha}\right)$ convergence rates. Thus our scheme gives a good approximation of the solution, interfaces, and coordinates of the hole-filling as predicted from Theorems 3.3 and 3.4 . 
Acknowledgements. This work was supported by the Portuguese FCT fellowship SFRH/BPD/88207/2012 and the UT Austin/Portugal CoLab project Phase Transitions and Free Boundary Problems.

\section{REFERENCES}

[1] L. Ambrosio, N. Fusco, and D. Pallara, Functions of bounded variation and free discontinuity problems. Oxford Math. Monogr. Clarendon Press, Oxford, New York (2000).

[2] D.G. Aronson, Regularity properties of flows through porous media: The interface. Arch. Rational Mech. Anal. 37 (1970) 1-10.

[3] D.G. Aronson, Regularity propeties of flows through porous media. SIAM J. Appl. Math. 17 (1969) 461-467.

[4] D.G. Aronson and P. Bénilan, Régularité des solutions de l'équation des milieux poreux dans $\mathbf{R}^{N}$. C. R. Acad. Sci. Paris Sér. $A-B 288$ (1979) A103-A105.

[5] L.A. Caffarelli, J.L. Vázquez, and N.I. Wolanski, Lipschitz continuity of solutions and interfaces of the $N$-dimensional porous medium equation. Indiana Univ. Math. J. 36 (1987) 373-401.

[6] L.A. Caffarelli and N. Wolanski, $C^{1, \alpha}$ regularity of the free boundary for the $N$-dimensional porous media equation. Commun. Pure Appl. Math. 43 (1990) 885-902.

[7] M.G. Crandall and M. Pierre, Regularizing effects for $u_{t}=\Delta \varphi(u)$. Trans. Amer. Math. Soc. 274 (1982) $159-168$.

[8] B.E.J. Dahlberg and C.E. Kenig, Nonnegative solutions of generalized porous medium equations. Rev. Mat. Iberoamericana 2 (1986) 267-305.

[9] P. Daskalopoulos and C.E. Kenig, Degenerate diffusions. Initial value problems and local regularity theory. Vol. 1 of EMS Tracts Math. European Mathematical Society (EMS), Zürich (2007).

[10] P. Daskalopoulos and E. Rhee, Free-boundary regularity for generalized porous medium equations. Commun. Pure Appl. Anal. 2 (2003) 481-494.

[11] A. de Pablo and J.L. Vázquez, Regularity of solutions and interfaces of a generalized porous medium equation in $\mathbf{R}^{N}$. Ann. Mat. Pura Appl. 158 (74) 51-74.

[12] F. del Teso and J.L. Vázquezn, Finite difference method for a general fractional porous medium equation. Preprint arXiv:1307.2474 (2013)

[13] E. DiBenedetto, Degenerate parabolic equations. Universitext. Springer-Verlag, New York (1993).

[14] E. DiBenedetto and D. Hoff, An interface tracking algorithm for the porous medium equation. Trans. Amer. Math. Soc. 284 (1984) 463-500.

[15] B.H. Gilding, Hölder continuity of solutions of parabolic equations. J. London Math. Soc. 13 (1976) 103-106.

[16] J.L. Graveleau and P. Jamet, A finite difference approach to some degenerate nonlinear parabolic equations. SIAM J. Appl. Math. 20 (223) 199-223.

[17] D. Hoff, A linearly implicit finite-difference scheme for the one-dimensional porous medium equation. Math. Comput. 45 (1985) 23-33.

[18] T. Nakaki and K. Tomoeda, A finite difference scheme for some nonlinear diffusion equations in an absorbing medium: support splitting phenomena. SIAM J. Numer. Anal. 40 (2002) 945-964.

[19] P.E. Sacks, Continuity of solutions of a singular parabolic equation. Nonlin. Anal. 7 (1983) 387-409.

[20] K. Tomoeda and M. Mimura, Numerical approximations to interface curves for a porous media equation. Hiroshima Math. J. 13 (1983) 273-294.

[21] K. Tomoeda, Numerically repeated support splitting and merging phenomena in a porous media equation with strong absorption. J. Math-Industry 3 (2011) 61-68.

[22] J.L. Vázquez, The porous medium equation. Oxford Math. Monogr. The Clarendon Press, Oxford University Press, Oxford (2007). Mathematical theory.

[23] Q. Zhang and Z. Wu, Numerical simulation for porous medium equation by local discontinuous Galerkin finite element method. J. Sci. Comput. 38 (2009) 127-148. 